\title{
Thermal-diffusion and diffusion-thermo effects on squeezing flow of unsteady magneto-hydrodynamic Casson fluid between two parallel plates with thermal radiation
}

\author{
N B NADUVINAMANI ${ }^{1, *}$ and USHA SHANKAR ${ }^{1,2}$ \\ ${ }^{1}$ Department of Mathematics, Gulbarga University, Kalaburagi 585 106, India \\ ${ }^{2}$ Department of Karnataka Power Corporation Limited, Raichur Thermal Power Station, \\ Shaktinagar, Raichur 584 170, India \\ e-mail: naduvinamaninb@yahoo.co.in
}

MS received 5 December 2018; revised 20 February 2019; accepted 22 May 2019

\begin{abstract}
Present numerical study examines the heat and mass transfer characteristics of unsteady magnetohydrodynamic squeezing flow of Casson fluid between two parallel plates with viscous and Joule dissipation effects in the presence of chemical reaction. The influence of Soret and Dufour parameters on squeezing flow is investigated along with thermal radiation and heat source/sink effects. The heat and mass transfer behaviour of squeezing flow is analysed by considering the rheological Casson fluid model. The present physical problem is governed by the set of nonlinear coupled time-dependent partial differential equations (PDEs). The method of similarity transformation approach is used to reduce the system of PDEs to a system of nonlinear ordinary differential equations (ODEs). Further, the Runge-Kutta fourth order integration scheme with shooting method (RK-SM) is used to solve the reduced ODEs. Numerical computations are performed for different sets of control parameters. The non-Newtonian flow behaviour of Casson fluid is presented in terms of graphs and tables. It is remarked that the temperature field is enhanced for increasing values of Hartmann number. Also, increasing Casson fluid parameter increases the velocity field. Concentration field is diminished for enhancing values of Soret parameter. Finally, the comparison between present similarity solutions and previously published results shows the accuracy of the current results.
\end{abstract}

Keywords. Soret and Dufour; Hartmann number; thermal radiation; Casson fluid; Joule dissipation; chemical reaction.

\section{Introduction}

Numerical heat and mass transfer characteristics of viscous fluid flow between two parallel disks/plates gained lot of attention in the current technology. This is owing to their large scale technical and industrial applications, like polymer processing, liquid metal lubrication, compression, squeezed film in power transmission and injection modelling. Most of the polymer processing cases, like thin sheets and paper formations, design of plastic and metal sheets, etc., include the squeezing flows. The literature [1-3] gives the best physical insight of these applications in detail. The biomedical applications comprise the flow inside syringes, nasogastric tubes, modelling of a synthetic materials or chemicals passage inside living bodies and physiological fluid flow through arteries due to the pumping of heart. Also, the synovial liquid inside the synovial cavity acts as a lubricant material between the hyaline cartilage or

*For correspondence

Published online: 08 July 2019 fibrocartilage, and the situation can be described through the squeezing phenomenon. However, there are some suitable examples of viscous non-Newtonian squeezing flows existing in bioengineering and biology [4-6].

The fundamental research work on squeezing flow was made by Stefan [7]. Based on the assumptions of lubrication theory, Stefan formulated the basic mathematical model for squeezing flow in detail under the appropriate thermodynamic conditions. Later a number of researchers gave their attention to study the thermodynamic behaviour of squeezing flows in different flow configurations. Reynolds [8] extended Stefan's problem to elliptic plates. Also, Archibald [9] studied a similar problem for rectangular plates. Since then, a number of scientists and engineers studied and analysed Stefan's work in various flow configurations in a better way [10-14]. Further, the heat and mass transfer behaviour of time-dependent viscous fluid flow between two parallel plates was studied by Mustafa et al [15] by using homotopy analysis method. Also, their study reports that, for the higher values of Prandtl and 
Eckert numbers, the temperature field enhances rapidly in the flow region.

The magneto-hydrodynamic (MHD) two-dimensional flow between two parallel plates was studied by Siddiqui et al [16] by using homotopy perturbation method (HPM). The investigation illustrated in [16] shows that increasing magnetic number diminishes the velocity field in the flow region. Domairry and Aziz [17] investigated a similar problem of squeezing flow of viscous incompressible fluid between two parallel disks, in which they presented the influence of inertia, magnetic field and injection/suction effects on flow behaviour. The influence of viscous dissipation on flow and heat transfer characteristics of unsteady two-dimensional viscous incompressible fluid between two impermeable parallel plates with constant temperature was studied by Duwairi et al [18]. It is noticed from their study that increasing squeezing number enhances the dimensional velocity field in the flow region. The flow sensitivity and thermal energy exchange of unsteady viscous incompressible fluid past a porous sensor surface kept in a squeezing channel with constant transpiration was studied by Mahmood et al [19]. It is observed from their study that increasing squeezing number decreases the velocity field. The steady-state axisymmetric squeezing flow of Newtonian fluid between parallel disks with heat transfer process was studied by Tashtoush et al [20]. The phenomenon of heat and mass transfer over a shrinking surface with suction effect was investigated by Muhamin et al [21]. The hydromagnetic time-independent flow and heat transfer characteristics of squeezed flow of electrically conducting viscous fluid between two parallel disks with finite-difference method was studied by Bahadir and Abbasov [22].

However, the phenomenon of heat transfer plays a key role in most of the industrial processes such as glass blowing, manufacturing of paper, spinning of metal, formation of glass fibres, coating of wires, hot rolling, aerodynamic extrusion of plastic sheets, continuous casting, drawing of plastic films, formation of glass sheets, etc. [1]. It is important to note that, in most of the cases, the temperature and concentration gradients occur in the energy and mass fluxes simultaneously. Consequently, the process of double diffusion becomes more complex in a moving fluid due to the coupling of heat and mass fluxes and the driving potentials. Further, the concentration gradients are also responsible for the generation of thermal flux in the moving fluid along with temperature gradients. Thus, the heat flux produced due to the presence of the concentration gradients is named as Dufour effect or diffusion-thermo effect. On other hand, mass flux can also be generated by the temperature gradients along with concentration gradients in the moving fluid and this effect is usually termed as thermal-diffusion or Soret effect. These effects have a key role in double diffusion phenomenon, where density difference occurs in the flow region. Clearly, Soret or thermaldiffusion effect is observed due to the presence of temperature gradients. On the other hand, Dufour or diffusion- thermo effect is produced due to the occurrence of concentration gradients in the moving fluid. Soret and Dufour effects cannot be ignored in case of gas mixture flows with lower molecular weights $\left(\mathrm{H}_{2}, \mathrm{He}\right)$ and greater molecular weights (air, $\mathrm{N}_{2}$ ).

Weaver and Viskanta [23] investigated the inter-diffusion of species and their effects along with thermal-diffusion and diffusion-thermo impacts on free-convection heat and mass transfer in cavities due to the combined concentration and temperature gradients. From their study it is observed that the flow is produced due to the temperature and concentration gradients in the flow regime. Raju and Sandeep [24] numerically analysed the heat and mass transfer characteristics of gyrotactic microorganisms suspended in a MHD Casson fluid flow near a vertical rotating plate or cone kept in porous medium. Sulochana et al [25] studied the Soret effect along with heat source or sink in case of three-dimensional Casson fluid flow over a stretching surface by accounting the heat and mass transfer characteristics. The influence of Dufour and Soret effects on electrically conducting time-independent MHD two-dimensional flow was studied by Nawaz et al [26]. The influence of the Soret and Dufour parameters on a channel flow of couple stress fluid with the chemical reaction and radiation effects was investigated by Ojjela and Kumar [27]. Also, Khan et al [28] studied the influence of Soret and Dufour effects, in case of viscous MHD flow between non-parallel walls by considering the chemical reaction process. From their study it is noticed that the temperature field increases with increasing Dufour number, and concentration field is diminished for increasing values of Soret number. Very recently, Khan et al [29] investigated the influence of Soret and Dufour effects on squeezing flow of MHD viscous incompressible nano-fluid between two parallel disks. Their study reports that, for the greater values of Schmidt and Soret numbers, the concentration field decreases in the flow region.

Having the above literature survey in consideration, it is important to note that, the present unsteady squeezing flow problem has large scale biological and engineering interest. Also, from the available literature it is noticed that, the squeezing behaviour of Casson fluid with MHD, Joule heating, thermal radiation and chemical reaction together with Soret and Dufour effects is paid less attention. With this research gap, authors have motivated and thus an attempt is made to investigate the heat and mass transfer behaviour of Casson fluid squeezing flow between two parallel plates by accounting the Soret and Dufour effects in the presence of chemical reaction and thermal radiation. However, this particular problem is not yet reported in the literature. The governing squeezing flow equations are obtained by considering the laws of fluid motion. The resulting coupled highly nonlinear flow equations are solved by using standard RK-SM and bvp4c methods. A detailed comparison between RK-SM and bvp4c technique is presented. Also, a comprehensive numerical discussion is made 
to describe the non-Newtonian flow behaviour of Casson fluid under the influence of various physical parameters.

\section{Non-Newtonian Casson fluid model}

The following rheological equation [30] is used to construct the governing equations for squeezing flow of Casson fluid between two parallel plates:

$$
\tau_{i j}=\left\{\begin{array}{cc}
2\left(\mu_{B}+\frac{P_{y}}{\sqrt{2 \pi}}\right) e_{i j}, & \pi>\pi_{c} \\
2\left(\mu_{B}+\frac{P_{y}}{\sqrt{2 \pi_{c}}}\right) e_{i j}, & \pi<\pi_{c}
\end{array}\right.
$$

In this model, the symbol $\pi=e_{i j} \cdot e_{i j}$ denotes the product of deformation components, $e_{i j}$ denotes the $(i, j)^{\text {th }}$ deformation rate component, $\pi_{c}$ is the critical value, $\mu_{B}$ is the dynamic plastic viscosity and $P_{y}$ is the yield stress.

\subsection{Mathematical formulation of the problem}

The present unsteady two-dimensional flow model considered the MHD radiative squeezing flow of viscous incompressible Casson fluid between two parallel plates with Soret and Dufour effects under the influence of chemical reaction process. Figure 1 clearly describes the geometry of the considered squeezing flow problem with necessary boundary conditions. In this model, the distance between two parallel plates is denoted by $y$ and it is taken as $\pm h(t)= \pm l(1-\alpha t)^{1 / 2}$, where $l$ denotes the initial position (when time $t=0$ ). Plates are squeezed when $\alpha>0$ till they reach $t=1 / \alpha$ and they are separated when $\alpha<0$. Soret and Dufour effects are considered in the concentration and temperature equations. Along with these effects, the Joule dissipation and heat generation due to frictional force produced by shear in flow are also accounted in the thermal equation. Also, the homogeneous first order chemical reaction effect is accounted in the concentration equation. Further, in the present analysis, symmetric flow is assumed. With these assumptions, the continuity, momentum, energy and concentration equations governing the present physical problem with necessary conditions are as follows [29-31]:

$$
\begin{gathered}
\frac{\partial u}{\partial x}+\frac{\partial v}{\partial y}=0 \\
\frac{\partial u}{\partial t}+u \frac{\partial u}{\partial x}+v \frac{\partial u}{\partial y}=-\frac{1}{\rho} \frac{\partial p}{\partial x} \\
+v\left(1+\frac{1}{\beta}\right)\left(2 \frac{\partial^{2} u}{\partial x^{2}}+\frac{\partial^{2} u}{\partial y^{2}}+\frac{\partial^{2} v}{\partial y \partial x}\right) \\
-\frac{\sigma B_{o}^{2}}{\rho(1-\alpha t)} u
\end{gathered}
$$

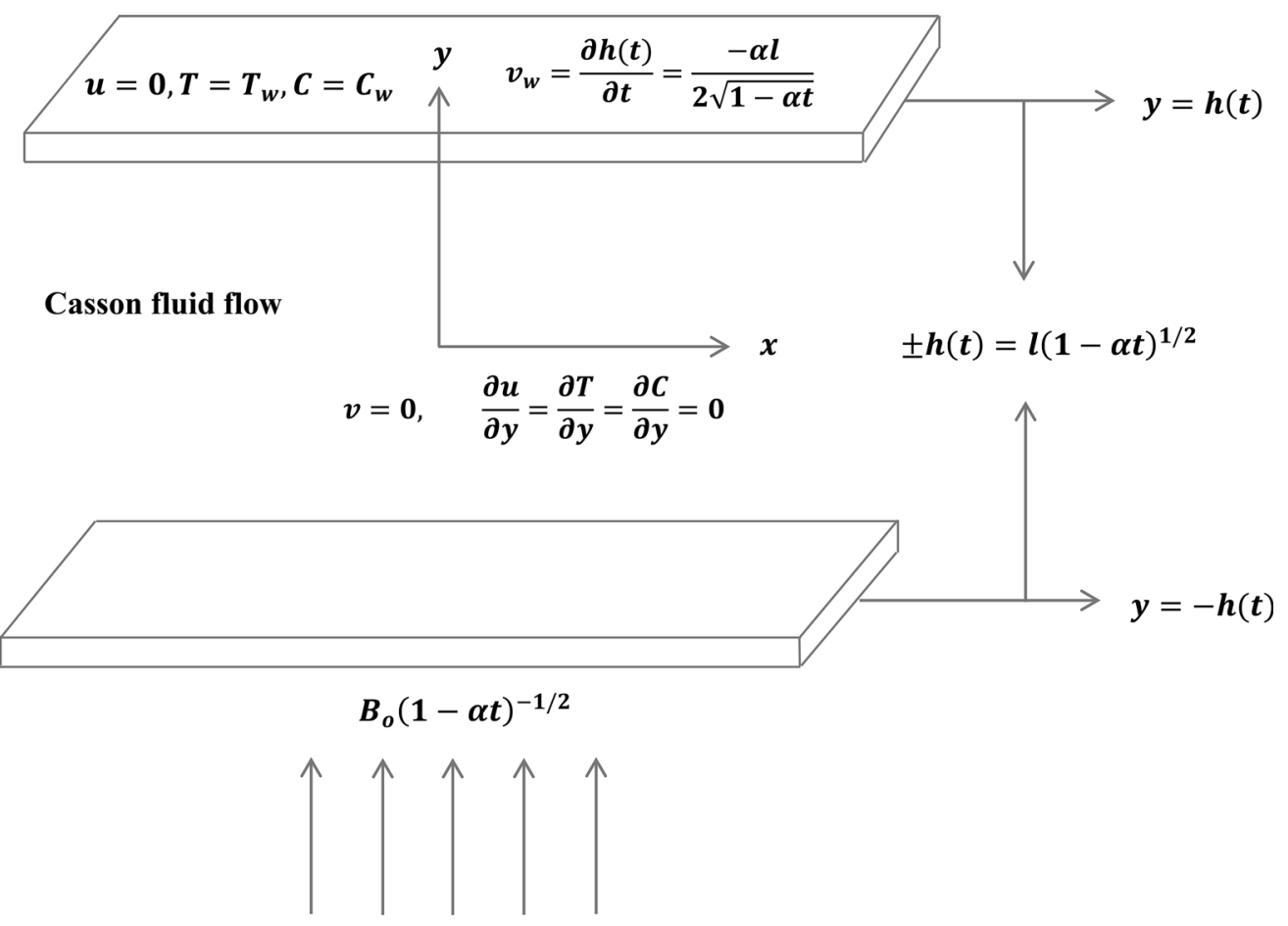

Figure 1. Flow configuration and coordinate system of the problem. 


$$
\begin{aligned}
& \frac{\partial v}{\partial t}+u \frac{\partial v}{\partial x}+v \frac{\partial v}{\partial y}=-\frac{1}{\rho} \frac{\partial p}{\partial y} \\
&+v\left(1+\frac{1}{\beta}\right)\left(2 \frac{\partial^{2} v}{\partial x^{2}}+\frac{\partial^{2} v}{\partial y^{2}}+\frac{\partial^{2} u}{\partial y \partial x}\right) \\
& \frac{\partial T}{\partial t}+u \frac{\partial T}{\partial x}+v \frac{\partial T}{\partial y}=\frac{k}{\rho c_{p}}\left(\frac{\partial^{2} T}{\partial x^{2}}+\frac{\partial^{2} T}{\partial y^{2}}\right) \\
&+\frac{\mu}{\rho c_{p}}\left(1+\frac{1}{\beta}\right)\left(2\left(\frac{\partial u}{\partial x}\right)^{2}+\left(\frac{\partial u}{\partial y}+\frac{\partial v}{\partial x}\right)^{2}+2\left(\frac{\partial v}{\partial y}\right)^{2}\right) \\
&+\frac{16 \sigma^{*} T_{o}^{3}}{3 \rho c_{p} k^{*}} \frac{\partial^{2} T}{\partial y^{2}}+\frac{\sigma B_{o}^{2}}{\rho c_{p}(1-\alpha t)} u^{2}+\frac{Q^{*}}{\rho C_{p}}\left(T-T_{\infty}\right) \\
&+\frac{D_{m} k_{T}}{c_{s} c_{p}} \frac{\partial^{2} C}{\partial y^{2}}
\end{aligned}
$$

$$
\begin{aligned}
\frac{\partial C}{\partial t}+u \frac{\partial C}{\partial x}+v \frac{\partial C}{\partial y}= & D_{m}\left(\frac{\partial^{2} C}{\partial x^{2}}+\frac{\partial^{2} C}{\partial y^{2}}\right)+\frac{D_{m} k_{T}}{T_{m}} \frac{\partial^{2} T}{\partial y^{2}} \\
& -\frac{k_{1}}{(1-\alpha t)}\left(C-C_{\infty}\right)
\end{aligned}
$$

The relevant boundary conditions for Casson fluid squeezing flow are given as follows:

$$
\begin{aligned}
& \begin{array}{l}
u=0, \quad v=v_{w}=\frac{d h}{d t}, \quad T=T_{w}, \quad C=C_{w}, \quad \text { at } \\
y=h(t),
\end{array} \\
& \frac{\partial u}{\partial y}=0, \quad v=0, \quad \frac{\partial T}{\partial y}=0, \quad \frac{\partial C}{\partial y}=0 \quad \text { at } \quad y=0 .
\end{aligned}
$$

In Eqs. (2)-(6), $u$ and $v$ are velocity components along $x$ and $y$ directions, respectively, $\rho$ is the density, $v$ is the kinematic viscosity, $\beta$ denotes the Casson fluid parameter, $\sigma$ is the electrical conductivity, $B_{o}$ is the magnetic field, $k$ denotes the thermal conductivity, $\alpha$ is the characteristic parameter of squeezing motion, $c_{p}$ is the specific heat capacity, $k_{T}$ is the thermal diffusion coefficient, $Q^{*}$ denotes the coefficient of heat source/sink, $T_{w}, C_{w}$ are the wall temperature and concentration, $T_{\infty}, C_{\infty}$ are the temperature and concentration of freestream, $k_{1}$ is the coefficient of chemical reaction and $D_{m}$ is the coefficient of mass diffusion.

\subsection{Similarity transformation approach}

Equations (2)-(6) with conditions (7a) and (7b) are unsteady, coupled and highly nonlinear in nature. Also, there are no analytical or direct methods available in literature to solve these coupled equations. Because of this reason, a standard numerical method namely, RK-SM is implemented. For this purpose, we first converted the set of coupled partial differential equations (PDEs) (2)-(6) to nonlinear ordinary differential equations (ODEs) using the following similarity transformations $[15,30,31]$ :

$$
\begin{gathered}
u=\left(\frac{\alpha x}{2(1-\alpha t)}\right) F^{\prime}(\eta), \quad v=\left(\frac{-\alpha l}{2 \sqrt{1-\alpha t}}\right) F(\eta), \\
\theta(\eta)=\frac{T-T_{\infty}}{T_{w}-T_{\infty}} \text { and } \phi(\eta)=\frac{C-C_{\infty}}{C_{w}-C_{\infty}}
\end{gathered}
$$

where $\eta=\frac{y}{l \sqrt{1-\alpha t}}$.

Using Eq. (8), the dimensional equations (2)-(6) are reduced to non-dimensional form as follows:

$$
\begin{aligned}
& \left(1+\frac{1}{\beta}\right) F^{\prime \prime \prime \prime}(\eta) \\
& -S\left(\eta F^{\prime \prime \prime}(\eta)+3 F^{\prime \prime}(\eta)+F^{\prime}(\eta) F^{\prime \prime}(\eta)-F(\eta) F^{\prime \prime \prime}(\eta)\right) \\
& -H a^{2} F^{\prime \prime}(\eta) \\
& =0
\end{aligned}
$$

$$
\begin{aligned}
& \left(1+\frac{4}{3} R\right) \theta^{\prime \prime}(\eta)+\operatorname{Pr} S\left(F(\eta) \theta^{\prime}(\eta)-\eta \theta^{\prime}(\eta)+Q \theta(\eta)\right) \\
& +\operatorname{Pr} E c\left(\left(1+\frac{1}{\beta}\right)\left(F^{\prime 2}(\eta)+4 \delta^{2} F^{\prime 2}(\eta)\right)+H a^{2} F^{\prime 2}(\eta)\right) \\
& +\operatorname{DuPr} \phi^{\prime \prime}(\eta)=0 \\
& \phi^{\prime \prime}(\eta)+\operatorname{Sc} S\left(F(\eta) \phi^{\prime}(\eta)-\eta \phi^{\prime}(\eta)\right)-\operatorname{ScKr} \phi(\eta) \\
& \quad+\operatorname{SrSc} \theta^{\prime \prime}(\eta) \\
& \quad=0 .
\end{aligned}
$$

Similarly, using Eq. (8), the dimensionless boundary conditions are obtained in the following form as

$$
\begin{aligned}
& F(\eta)=0, \quad F^{\prime \prime}(\eta)=0, \quad \theta^{\prime}(\eta)=0, \quad \phi^{\prime}(\eta)=0 \quad \text { at } \\
& \eta=0 \text {, } \\
& F(\eta)=1, \quad F^{\prime}(\eta)=0, \quad \theta(\eta)=1, \quad \phi(\eta)=1 \quad \text { at } \\
& \eta=1 \text {. }
\end{aligned}
$$

In Eqs. (9)-(12), the superscript symbol prime denotes differentiation with respect to similarity variable $\eta$. Also, the non-dimensional physical parameters in Eqs. (9)-(11) are defined as follows:

$S=\frac{\alpha l^{2}}{2 v}$ (squeezing number), $R=\frac{4 \sigma^{*} T_{\infty}^{3}}{k k^{*}}$ (radiation parameter), $H a=B_{o} l \sqrt{\frac{\sigma}{\mu}}$ (Hartmann number), Du= $\frac{D_{m} k_{T}\left(C-C_{\infty}\right)}{c_{s} c_{p} v\left(T-T_{\infty}\right)}$ (Dufour number), $S r=\frac{D_{m} k_{T}\left(T-T_{\infty}\right)}{T_{m} v\left(C-C_{\infty}\right)}$ (Soret number), $S c=\frac{v}{D_{m}}$ (Schmidt number), $\operatorname{Pr}=\frac{\mu C_{p}}{k}$ (Prandtl number), $\delta=\frac{h}{x}, K r=$ $\frac{k_{1} l^{2}}{v}$ (chemical reaction parameter), $Q=\frac{2 Q^{*}(1-\alpha t)}{\alpha \rho C_{p}}$ (heat source/sink parameter) and $E c=\frac{\alpha^{2} x^{2}}{4 C_{p}\left(T_{w}-T_{\infty}\right)(1-\alpha t)^{2}}$ (Eckert number). 
The movement of plates is completely described with the help of squeezing number ( $S$ ) (i.e., $S>0$ is separation of plates from one another; $S<0$ is movement of plates close to one another, and this type of flow is identified as method begins by discretizing the coupled higher order ODEs into a set of first order ODEs. Following are the required first order ODEs used in the numerical computations:

$$
\begin{gathered}
\frac{d F_{0}}{d \eta}=F_{1}, \\
\frac{d F_{1}}{d \eta}=F_{2}, \\
\frac{d F_{2}}{d \eta}=F_{3}, \\
\frac{d F_{3}}{d \eta}=\left(\frac{1}{1+\frac{1}{\beta}}\right)\left(S\left(\eta F_{3}(\eta)+3 F_{2}(\eta)+F_{1}(\eta) F_{2}(\eta)-F_{0}(\eta) F_{3}(\eta)\right)+2 H a^{2} F_{2}(\eta)\right), \\
\frac{d \theta_{1}}{d \eta}=\left(\begin{array}{c}
\frac{-1}{d \eta} \\
1+\frac{4}{3} R
\end{array}\right)\left(\begin{array}{c}
\operatorname{Pr} E c\left(\left(1+\frac{1}{\beta}\right)\left(F_{2}^{2}(\eta)+4 \delta^{2} F_{1}^{2}(\eta)\right)+H a^{2} F_{1}^{2}(\eta)\right) \\
\left.+\operatorname{Pr} S\left(F_{0}(\eta) \theta_{1}(\eta)-\eta \theta_{1}(\eta)+Q \theta_{0}\right)++\operatorname{DuPr} \frac{d \phi_{1}}{d \eta}\right), \\
\frac{d \phi_{0}}{d \eta}=\phi_{1}, \\
\frac{d \phi_{1}}{d \eta}=-\left(\operatorname{ScS}\left(F_{0}(\eta) \phi_{1}(\eta)-\eta \phi_{1}(\eta)\right)-S c K r \phi_{0}(\eta)+S r S c \frac{d \theta_{1}}{d \eta}\right) .
\end{array}\right)
\end{gathered}
$$

squeezing flow). Velocity field is regulated with the help of Hartmann number. Similarly, to control the temperature field, Prandtl and Eckert numbers are useful. Additionally, for $E c=0$ viscous dissipation effects are absent. Further, Dufour and Soret effects are described in temperature and concentration equations. In this analysis $K r<0, K r>0$ and $K r=0$ correspond to destructive, constructive and no chemical reactions, respectively. Also, Schmidt number is used to control the concentration field in the flow region. In the present investigation, Runge-Kutta fourth order integration scheme with shooting technique is used to produce the approximate numerical similarity solution for all the non-dimensional parameters. To this end, the following numerical solution procedure is described.

\section{Numerical solution scheme}

As mentioned earlier, the set of equations governing the time-dependent MHD Casson fluid flow between parallel plates with thermal-diffusion and diffusion-thermo effects are nonlinear and coupled in nature. Thus, to solve these coupled flow equations (9)-(11) with conditions (12), a numerically stable Runge-Kutta fourth order integration scheme with standard shooting technique [32] is used. This
Also, it is considered that $F(\eta)=F_{0}(\eta), \theta(\eta)=\theta_{0}(\eta)$ and $\phi(\eta)=\phi_{0}(\eta)$. With these assumptions, the following initial and boundary conditions are used to solve Eq. (13):

$$
\left.\begin{array}{c}
F_{0}(\eta)=0, \quad F_{2}(\eta)=0, \quad \text { at } \eta=0 \\
F_{0}(\eta)=1, \quad F_{1}(\eta)=0 \quad \text { at } \eta=1
\end{array}\right\},
$$

However, in order to solve this modified system of first order ODEs (13), using the Runge-Kutta method, eight initial conditions are compulsory, whereas only four $\left(F_{0}(0)\right.$, $F_{2}(0), \theta_{1}(0)$ and $\left.\phi_{1}(0)\right)$ of them are given. Four more initial conditions are required to replace the boundary conditions $F_{0}(\eta)=1, F_{1}(\eta)=0, \theta_{0}(\eta)=1$ and $\phi_{0}(\eta)=1$ defined at $\eta=1$. Thus, it is assumed that $F_{0}(0)=m_{1}$, $F_{1}(0)=m_{2}, \theta_{0}(0)=m_{3}$ and $\phi_{0}(0)=m_{4}$ are the required initial conditions. The unknown values $m_{1}, m_{2}, m_{3}$ and $m_{4}$ of these initial conditions are first predicted and consequently resolved using the Newton-Raphson method for individual parameters with respect to the given boundary conditions. These values are modified such that the 
Table 1. Comparison of momentum, heat and mass transport coefficients, between present results (RK-SM) and analytical (HAM) results obtained by Mustafa et al [15] for different values of $S$ when $H a \rightarrow 0, R \rightarrow 0, Q \rightarrow 0, D u \rightarrow 0, S r \rightarrow 0, \beta \rightarrow \infty$ and $\eta=1$.

\begin{tabular}{|c|c|c|c|c|c|c|}
\hline \multirow[b]{2}{*}{ Squeezing number $S$} & \multicolumn{3}{|c|}{ Mustafa et al [15] } & \multicolumn{3}{|c|}{ Present results } \\
\hline & $-F^{\prime \prime}(1)$ & $-\theta^{\prime}(1)$ & $-\phi^{\prime}(1)$ & $-F^{\prime \prime}(1)$ & $-\theta^{\prime}(1)$ & $-\phi^{\prime}(1)$ \\
\hline-1.0 & 2.170090 & 3.319899 & 0.8045587 & 2.170090 & 3.319899 & 0.8045587 \\
\hline-0.5 & 2.614038 & 3.129491 & 0.7814023 & 2.614038 & 3.129491 & 0.7814023 \\
\hline 0.01 & 3.007134 & 3.047092 & 0.7612252 & 3.007134 & 3.047092 & 0.7612252 \\
\hline 0.5 & 3.336449 & 3.026324 & 0.7442243 & 3.336449 & 3.026324 & 0.7442243 \\
\hline 2.0 & 4.167389 & 3.118551 & 0.7018132 & 4.167389 & 3.118551 & 0.7018132 \\
\hline
\end{tabular}

Table 2. Convergence test results obtained based on RK-SM and bvp4c techniques for $S=H a=\delta=K r=0.5, P r=S c=1.5, D u=$ $S r=0, \beta=R=Q=E c=0.3$ and $\eta=1$.

\begin{tabular}{|c|c|c|c|c|c|c|}
\hline \multirow[b]{2}{*}{$\eta$} & \multicolumn{2}{|c|}{$F(\eta)$} & \multicolumn{2}{|c|}{$\theta(\eta)$} & \multicolumn{2}{|c|}{$\phi(\eta)$} \\
\hline & RK-SM & bvp4c & RK-SM & bvp4c & RK-SM & bvp4c \\
\hline 0.0 & 0.000000 & 0.000000 & 3.40651 & 3.40650 & 0.722235 & 0.722235 \\
\hline 0.1 & 0.148340 & 0.148340 & 3.38743 & 3.38742 & 0.724944 & 0.724944 \\
\hline 0.2 & 0.293821 & 0.293821 & 3.32979 & 3.32978 & 0.733070 & 0.733070 \\
\hline 0.3 & 0.433566 & 0.433566 & 3.23236 & 3.23236 & 0.746619 & 0.746619 \\
\hline 0.4 & 0.564672 & 0.564672 & 3.09287 & 3.09286 & 0.765606 & 0.765606 \\
\hline 0.5 & 0.684191 & 0.684191 & 2.90768 & 2.90767 & 0.790070 & 0.790070 \\
\hline 0.6 & 0.789116 & 0.789116 & 2.67132 & 2.67131 & 0.820089 & 0.820089 \\
\hline 0.7 & 0.876369 & 0.876369 & 2.37586 & 2.37586 & 0.855800 & 0.855800 \\
\hline 0.8 & 0.942791 & 0.942791 & 2.01011 & 2.01011 & 0.897429 & 0.897429 \\
\hline 0.9 & 0.985122 & 0.985122 & 1.55855 & 1.55855 & 0.945323 & 0.945323 \\
\hline 1.0 & 1.000000 & 1.000000 & 1.00000 & 1.00000 & 1.000000 & 1.000000 \\
\hline
\end{tabular}

boundary conditions are satisfied for $\eta=1$. The resulting initial value problem is attempted numerically using fourth order Runge-Kutta integration scheme. The convergence criterion and step size are taken, respectively, to be $10^{-5}$ and 0.01 for all numerical solutions. However, once the convergence criterion is approached, the system of ODEs are assimilated using the Runge-Kutta method to obtain the required results in terms flow profiles. More details about the Runge-Kutta method can be found in the available literature [33].

\section{Results and discussion}

\subsection{Verification of present numerical results}

The accuracy of Runge-Kutta scheme with shooting method (RK-SM) and obtained similarity solutions are verified by comparing them with the semi-analytical results of Mustafa et al [15]. This comparison is shown in table 1. Further, from table 1 it is noted that, the absolute value of wall shear stress is magnified for enhancing values of squeezing number whereas Sherwood and Nusselt numbers decrease. Also, it is clear that, the negative values of Nusselt number indicate flow of heat from plates to the ambient fluid. Further, the accuracy and correctness of RK-SM are illustrated with the help of table 2. From table 2 it is noticed that, the numerical results obtained based on RK-SM show the excellent agreement with the results obtained based on bvp $4 \mathrm{c}$ method. Further, this comparison clearly demonstrates the consistency of RKSM in obtaining the solution of highly nonlinear problems. The detailed literature on stability and convergence of Runge-Kutta method can found in the available literature [34-38].

\subsection{Influence of control parameters on flow behaviour}

The physical insight of the present unsteady squeezing problem is well described by analysing the effects of various control parameters on flow, heat and mass transfer behaviour. The physics behind this particular problem is illustrated in terms of flow profiles for the different values of control parameters such as $S, R, \beta, P r, H a, K r, D u, Q$, $E c, S r$ and $S c$. Also, for the better understanding of similarity results, computer-generated numerical data is expressed in terms of tables and graphs.

(i) Effect of squeezing number $(S)$ on flow profiles

The thermodynamic variations noticed in flow profiles under the influence of squeezing number $(S)$ are depicted in figures 2, 3, 4 and 5. In the present study, $S>0$ corresponds to the moment when the plates are away from one 


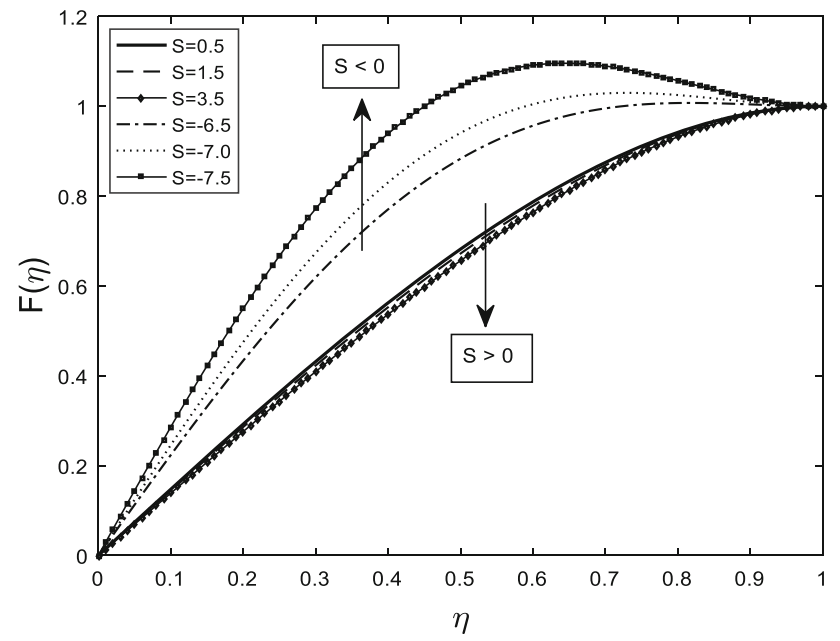

Figure 2. Effect of $S$ on $F(\eta)$ for fixed $\beta=0.8, H a=0.1, D u=$ $0.5, R=Q=P r=E c=K r=0.1, S c=0.7, S r=0.1$ and $\delta=5$.

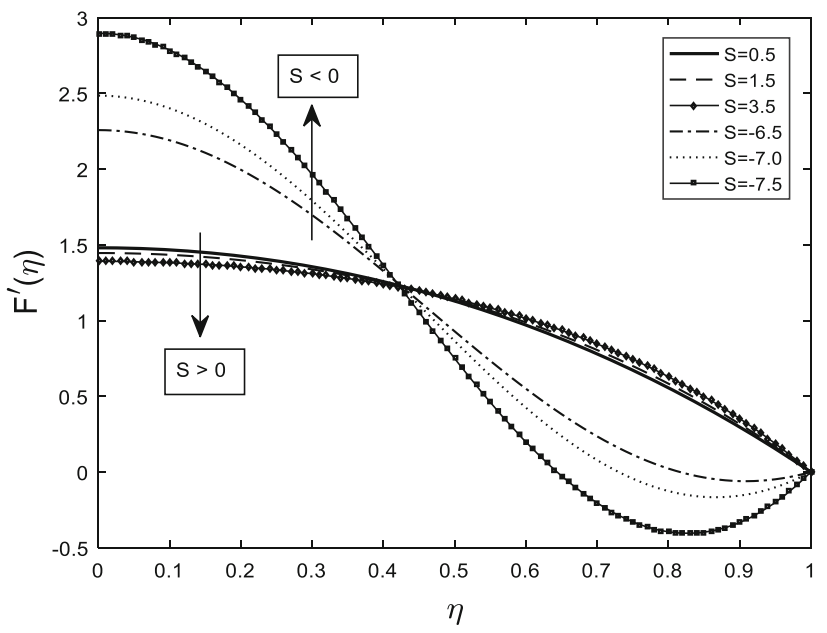

Figure 3. Impact of $S$ on $F^{\prime}(\eta)$ for fixed $\beta=0.8, H a=$ $0.1, D u=0.5, R=Q=P r=E c=K r=0.1, S c=0.7, S r=0.1$ and $\delta=5$.

another and $S<0$ corresponds to the moment when the plates are close to another. Figure 2 illustrates that the normal velocity component suppressed in the region $0<\eta<1$ for $S>0$ and it is enhanced for $S<0$. This is due to the fact that, when plates move apart, the fluid is sucked into the channel, which gives increased velocity in the channel. On the other hand, when plates move close to one another, the liquid inside the channel is released out into the channel, which produces the liquid drop inside the channel and hence velocity of the fluid decays.

The axial velocity profile $\left(F^{\prime}\right)$ is illustrated in figure 3 . Figure 3 depicts that $F^{\prime}$ decreases in the region $\eta<\eta_{c}$ and it increases in the region $\eta_{c}<\eta$ for $S>0$, where $\eta_{c}=0.45$. Also, from figure 3 it is remarked that, $F^{\prime}$ is magnified in the region $\eta<\eta_{c}$ and it decreases in the region $\eta_{c}<\eta$ for $S<0$, where $\eta_{c}=0.45$. Also, it is observed that, at the

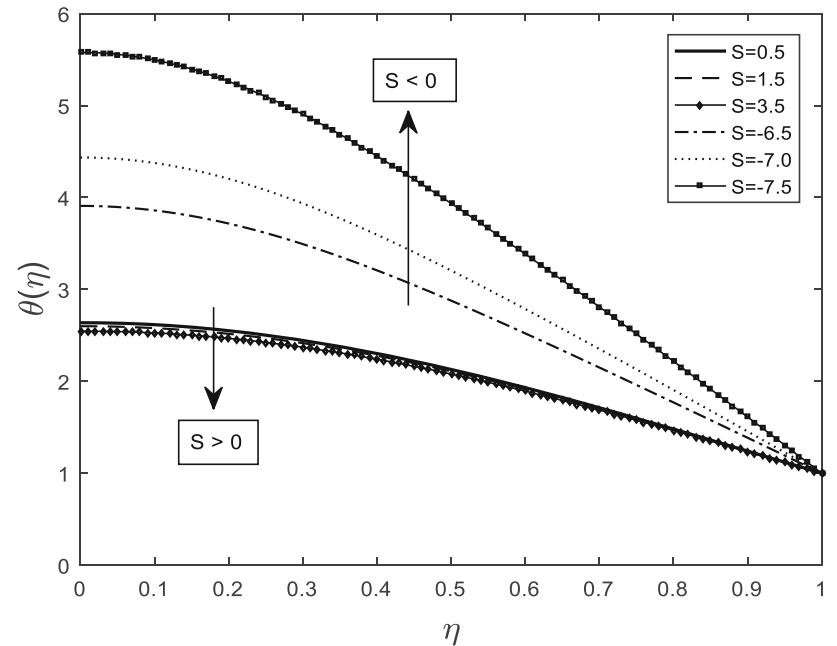

Figure 4. Effect of $S$ on $\theta(\eta)$ for fixed $\beta=0.8, H a=0.1, D u=$ $0.5, R=Q=P r=E c=K r=0.1, S c=0.7, S r=0.1$ and $\delta=5$.

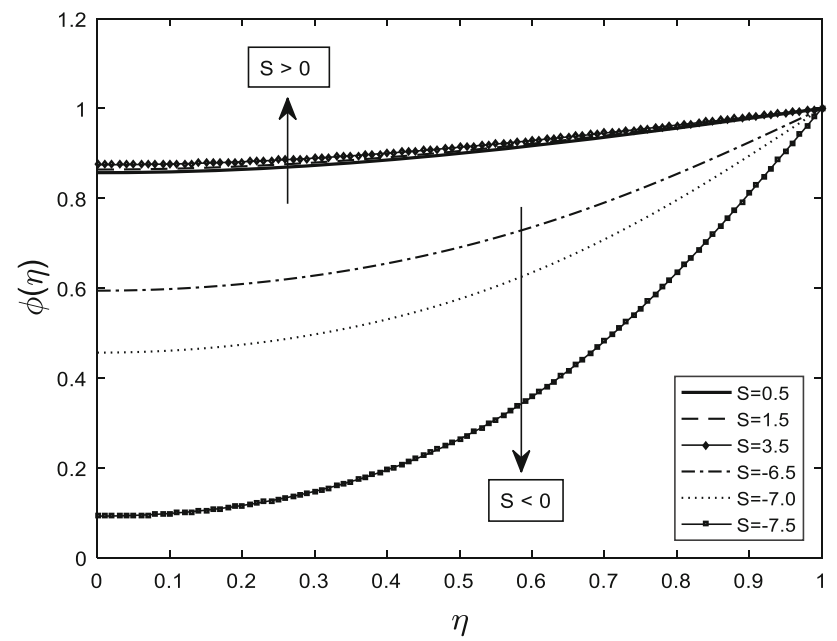

Figure 5. Impact of $S$ on $\phi(\eta)$ for fixed $\beta=0.8, H a=$ $0.1, D u=0.5, R=Q=P r=E c=K r=0.1, S c=0.7, S r=0.1$ and $\delta=5$.

critical point $\eta=0.45$, all the velocity curves coincide, which indicates that the squeezing number has the same effect on velocity field in the flow region. Owing to these changes in axial velocity at the boundaries, cross-flow behaviour is noticed at the central portion of the channel.

The influence of $S$ on temperature profile $(\theta)$ is depicted in figure 4 . Figure 4 illustrates that the $\theta$ profile decreases for $S>0$, and it increases for $S<0$. The decrease in the temperature field is due to the increased length between the plates, which decreases kinematic viscosity of the fluid, which in turn magnifies the speed of the plates and hence decreases the temperature field.

The effect of $S$ on concentration field $(\phi)$ is illustrated in figure 5. It is noticed from figure 5 that the concentration field increases for the increasing values of $S>0$ and it decreases for the decreasing values of $S<0$ in the flow 


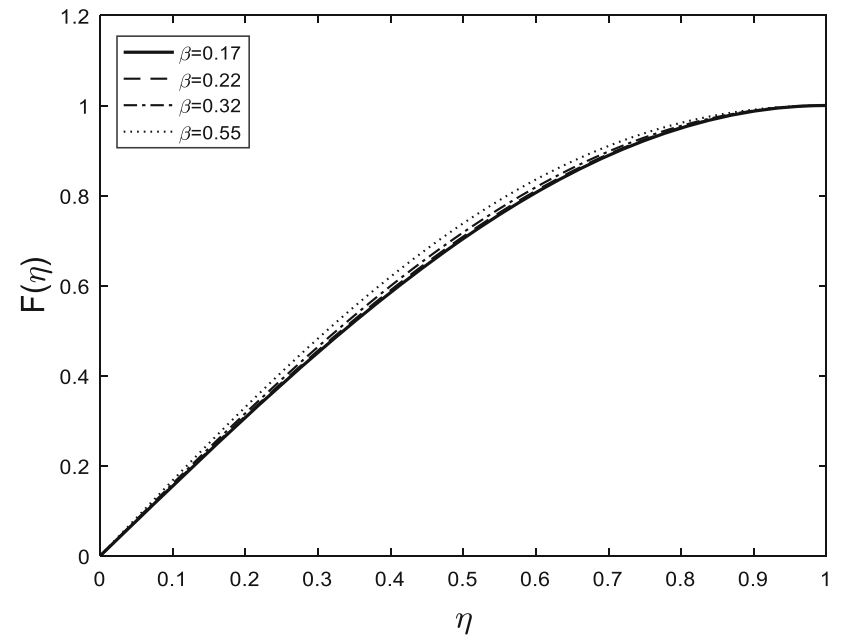

Figure 6. Influence of $\beta$ on $F(\eta)$ for fixed $S=-4, H a=D u=$ $0.1, S r=0.5, R=Q=K r=E c=0.1, P r=S c=0.7$ and $\delta=1.2$.

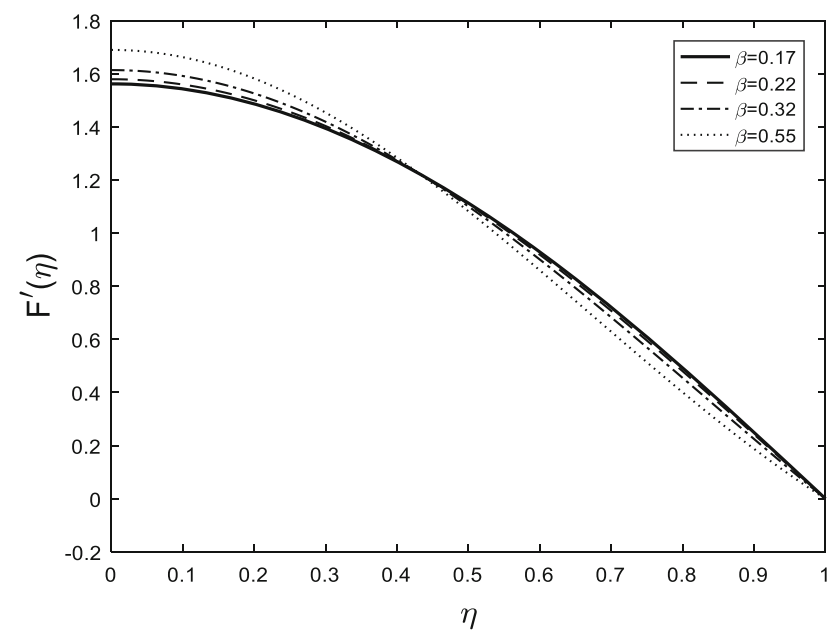

Figure 7. Impact of $\beta$ on $F^{\prime}(\eta)$ for fixed $S=-4, H a=D u=$ $0.1, S r=0.5, R=Q=K r=E c=0.1, P r=S c=0.7$ and $\delta=$ 1.2 .

region. In the neighbourhood of upper plate (i.e., $\eta \approx 1$ ), all the $\phi$ curves are merged, which shows that $S$ has no significant effect on $\phi$ profile near the upper plate when compared with the region close to the lower plate.

(ii) Influence of Casson fluid parameter $(\beta)$ on flow profiles

Figures 6, 7, 8 and 9 illustrate the effect of Casson fluid parameter $(\beta)$ on flow field variables. From figure 6 it is noticed that, as $\beta$ increases, the normal velocity field increases. This is because under the effect of applied stresses the small increment in $\beta$ decreases fluid viscosity and hence this decreased viscosity offers less resistance to the flow of fluid in the channel. Further, it is observed that, in the vicinity of lower and upper plates, $\beta$ has less effect when compared with other regions of the channel. Figure 7 illustrates the effect of $\beta$ on axial velocity profile. It is

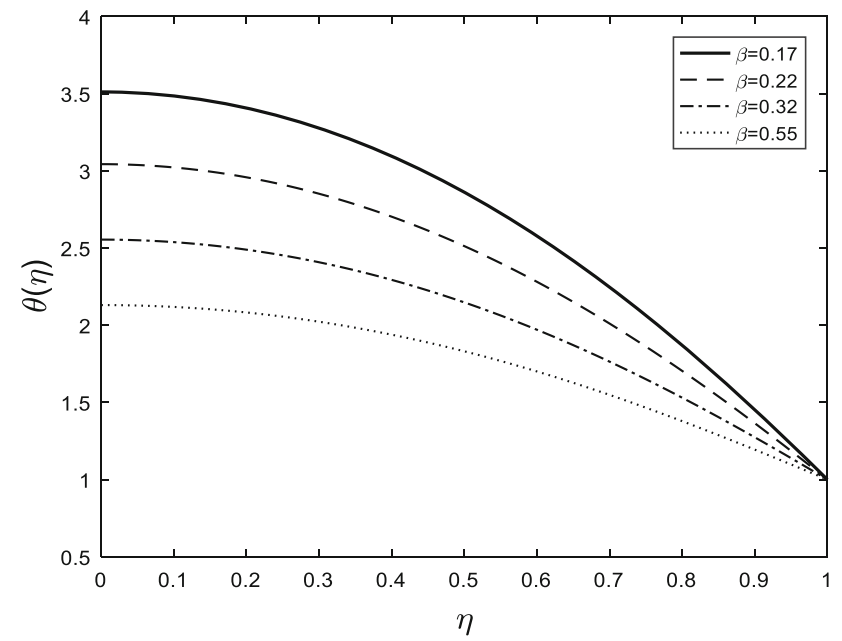

Figure 8. Effect of $\beta$ on $\theta(\eta)$ for fixed $S=-4, H a=D u=0.1$, $S r=0.5, R=Q=K r=E c=0.1, P r=S c=0.7$ and $\delta=1.2$.

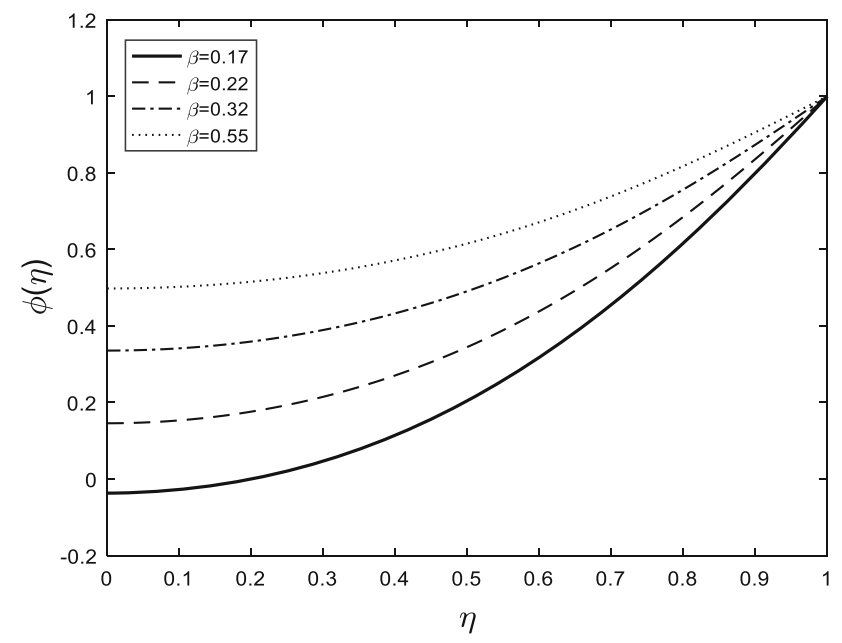

Figure 9. Impact of $\beta$ on $\phi(\eta)$ for fixed $S=-4, H a=D u=$ $0.1, S r=0.5, R=Q=K r=E c=0.1, P r=S c=0.7$ and $\delta=$ 1.2 .

noticed from this figure that $F^{\prime}$ increases in the region $\eta<\eta_{c}$ and it decreases in the remaining portion $\eta_{c}<\eta$ for the magnifying $\beta$ values, where $\eta_{c}=0.45$. Due to these variations in axial velocity at the boundaries, very interesting cross-flow behaviour is noticed at the central portion of the channel.

Similarly, the effect of $\beta$ on $\theta$ profile is described through figure 8 . It is noticed from this figure that temperature field decreases as $\beta$ increases. However, the effect of $\beta$ on $\phi$ profile is depicted in figure 9. Also, figure 9 shows that, as $\beta$ increases, the concentration field increases. Clearly, concentration field is an increasing function of $\beta$.

(iii) Impact of $\mathrm{Ha}$ on flow behaviour

The impact of $\mathrm{Ha}$ on flow profiles is depicted in figures 10, 11, 12 and 13. Figure 10 illustrates the effect of $\mathrm{Ha}$ on normal velocity field $(F)$ in the flow region. It is noticed 


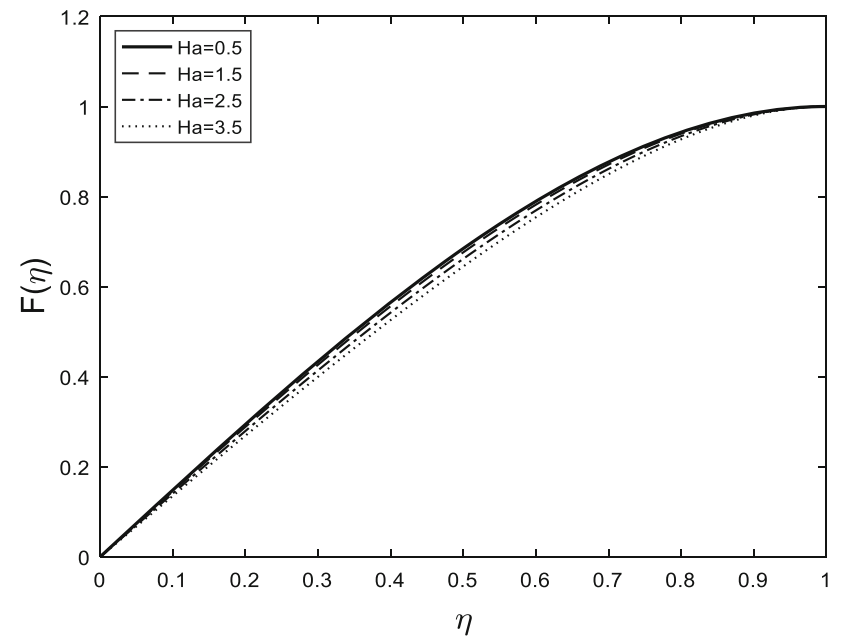

Figure 10. Influence of $H a$ on $F(\eta)$ for fixed $S=0.1, \beta=$ 2.0, $D u=S r=0.5, R=Q=E c=K r=0.1, P r=S c=0.7$ and $\delta=0.5$.

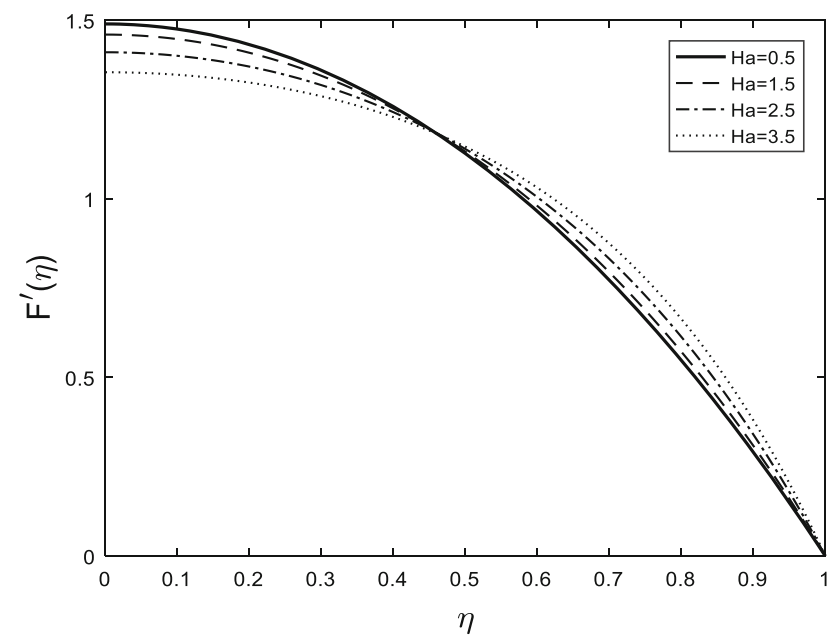

Figure 11. Effect of $H a$ on $F^{\prime}(\eta)$ for fixed $S=0.1, \beta=$ 2.0, $D u=S r=0.5, R=Q=E c=K r=0.1, P r=S c=0.7$ and $\delta=0.5$.

from figure 10 that the normal velocity field decays as $\mathrm{Ha}$ increases. This is due to the presence of stronger Lorentz forces in the flow region; these forces offer more opposition to the flow of fluid inside the channel and hence velocity field decreases. Figure 11 shows the effect of $\mathrm{Ha}$ on axial velocity $F^{\prime}$ profile. It is remarked from this figure that the axial velocity decreases in the region $\eta<\eta_{c}$ and it increases in the remaining portion $\eta_{c}<\eta$ of the channel, where $\eta_{c}=0.45$. Further, at the point $\eta \approx 0.46$, the effect of $H a$ is almost same.

The influence of $H a$ on $\theta$ profile is portrayed in figure 12 . It is observed from figure 12 that, as $\mathrm{Ha}$ increases, the thermal field eventually increases. This is due to the presence of Joule and viscous dissipation effects in the thermal equation. Further, the effect of $\mathrm{Ha}$ on concentration profile

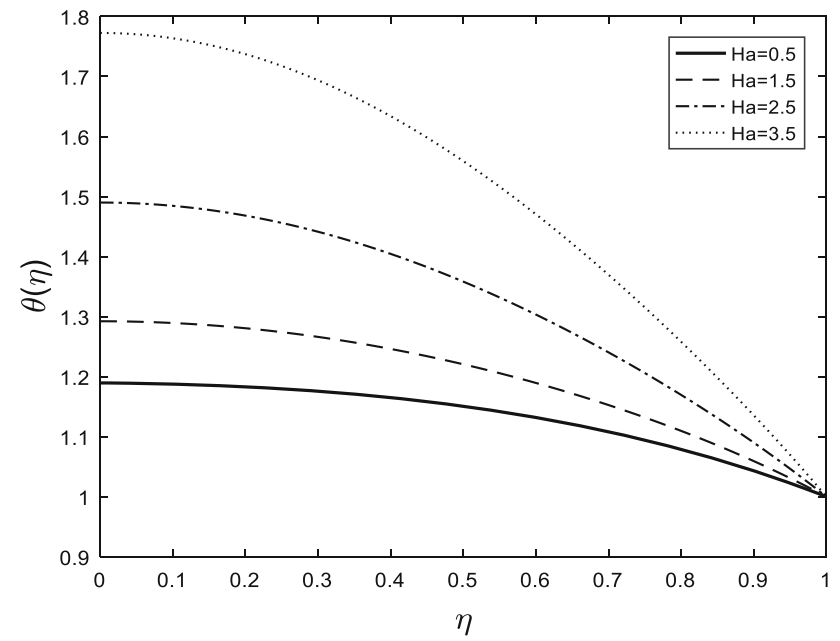

Figure 12. Impact of $H a$ on $\theta(\eta)$ for fixed $S=0.1, \beta=$ 2.0, $D u=S r=0.5, R=Q=E c=K r=0.1, P r=S c=0.7$ and $\delta=0.5$.

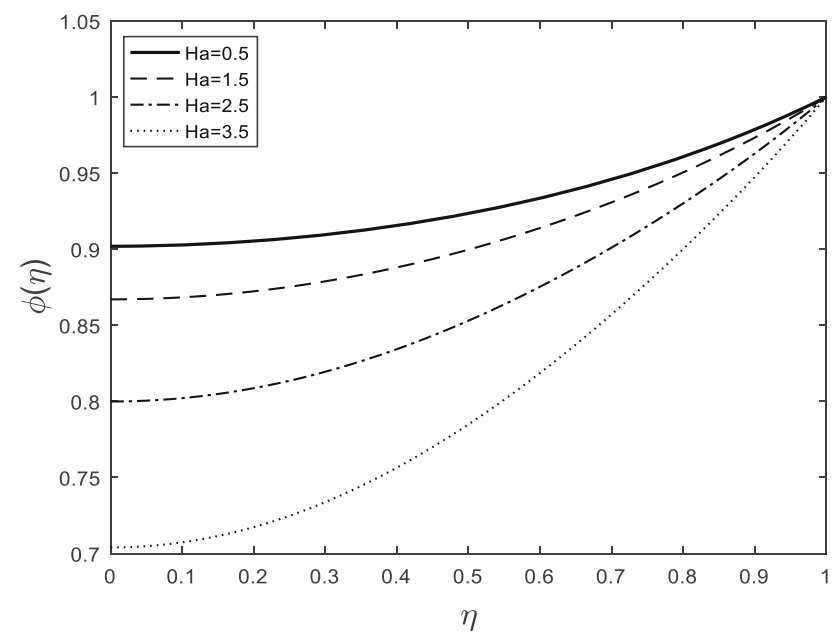

Figure 13. Effect of $H a$ on $\phi(\eta)$ for fixed $S=0.1, \beta=$ 2.0, $D u=S r=0.5, R=Q=E c=K r=0.1, P r=S c=0.7$ and $\delta=0.5$.

is portrayed in figure 13. Clearly, figure 13 indicates that, as $\mathrm{Ha}$ increases, concentration field decreases in the flow region.

(iv) Influence of radiation parameter $(R)$ on $\theta$ and $\phi$ profiles

The impact of $R$ on $\theta$ and $\phi$ profiles is illustrated in figures 14 and 15, respectively. Figure 14 depicts that temperature field decays for increasing values of $R$. This is because, as per the relation $R=\frac{4 \sigma^{*} T_{0}^{3}}{\kappa k^{*}}$, an increment in $R$ decreases the absorption coefficient $k^{*}$, which decreases the temperature field in the flow region. Therefore, these results are reasonable and acceptable. Physically, an upsurge in $R$ causes higher temperature values, which may be advantageous in the thermodynamic industries. Further, the effect of $R$ on $\phi$ field is described in figure 15. The $\phi$ profile 


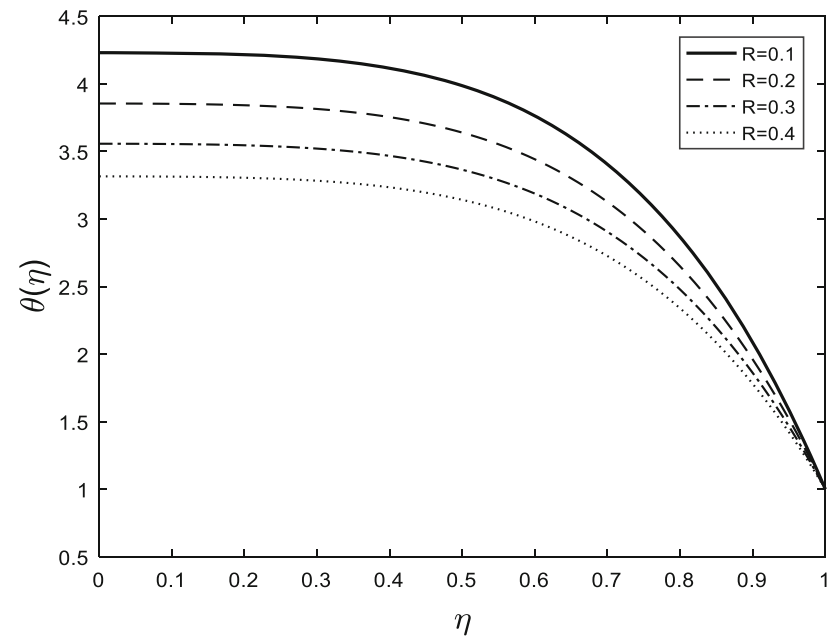

Figure 14. Influence of $R$ on $\theta(\eta)$ for fixed $S=0.4, \beta=$ $0.2, D u=S r=0.5, H a=Q=K r=0.1, E c=1.0, P r=S c=0.7$ and $\delta=0.1$.

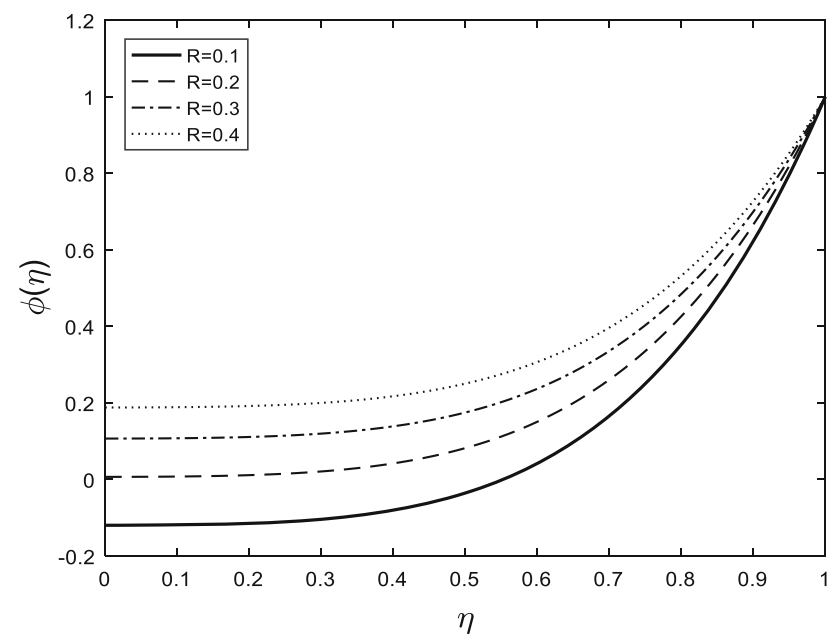

Figure 15. Impact of $R$ on $\phi(\eta)$ for fixed $S=0.4, \beta=0.2, D u=$ $S r=0.5, H a=Q=K r=0.1, E c=1.0, P r=S c=0.7$ and $\delta=$ 0.1 .

shows the increasing trend for increasing values of thermal radiation parameter, which is shown in figure 15 .

(v) Impact of $Q$ on temperature and concentration profiles

Figures 16 and 17 depict the effect of heat generation or absorption parameter $(Q)$ on $\theta$ and $\phi$ fields, respectively. Figure 16 demonstrates that, as $Q$ increases the thermal field increases and for $Q<0$, temperature profile decreases. This is because temperature of the working fluid increases during the heat generation process. Due to this reason, the thermal field is magnified in the flow region. Also, exothermic chemical reaction may increase temperature field. Additionally, figure 17 describes the effect of $Q$ on concentration profile. From this figure, it is noticed that, as $Q$ increases negatively, the concentration field increases in

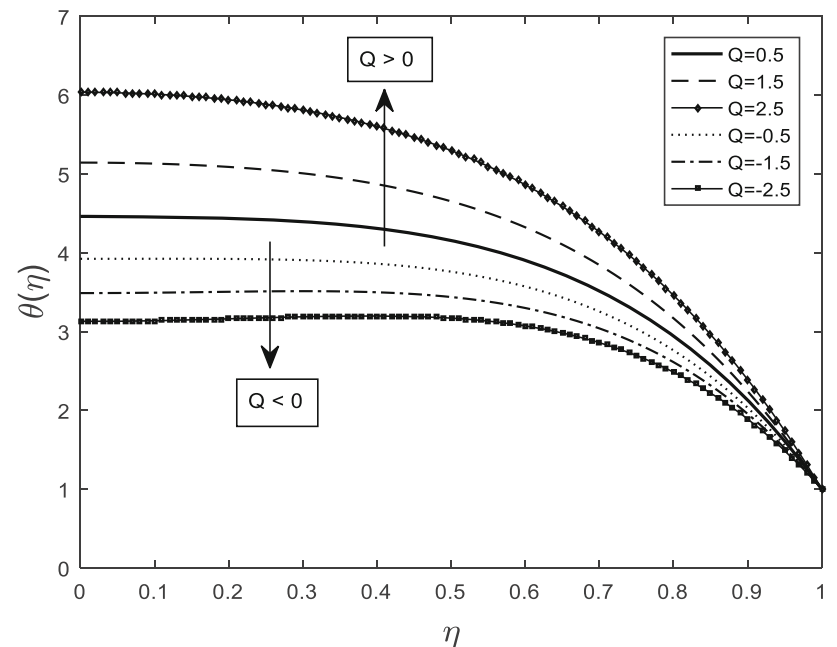

Figure 16. Effect of $Q$ on $\theta(\eta)$ for fixed $S=0.4, \beta=0.2, D u=$ $S r=0.5, H a=R=K r=0.1, E c=1.0, P r=S c=0.7$ and $\delta=$ 0.1 .

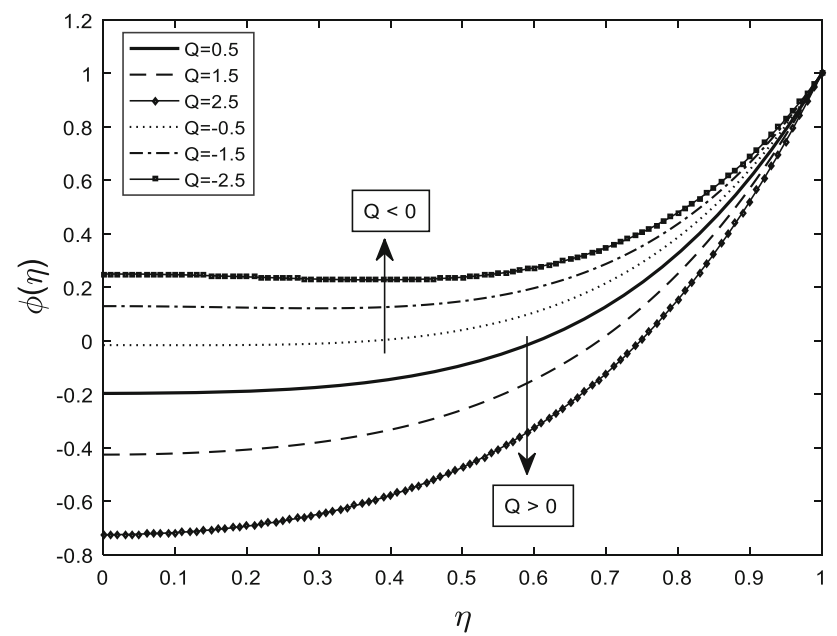

Figure 17. Impact of $Q$ on $\phi(\eta)$ for fixed $S=0.4, \beta=0.2, D u=$ $S r=0.5, H a=R=K r=0.1, E c=1.0, P r=S c=0.7$ and $\delta=$ 0.1 .

the flow region and the concentration field decreases for the positively increasing values of $Q>0$.

(vi) Influence of $E c$ on $\theta$ and $\phi$ profiles

The effect of $E c$ on $\theta$ and $\phi$ profiles is illustrated, respectively, in figures 18 and 19. Figures 18 and 19 demonstrate that, as $E c$ increases, the temperature profile increases and concentration profile decreases. This is due to the fact that the presence of frictional forces in the fluid causes release of heat energy into the fluid, which magnifies the temperature field in the flow region. Additionally, presence of viscous dissipation also increases the temperature field [13]. It is noticed that $E c$ explicitly occurs in the temperature equation, and hence temperature field can be easily regulated by controlling $E c$. Further, the concentration profile decreases for increasing values of $E c$. 


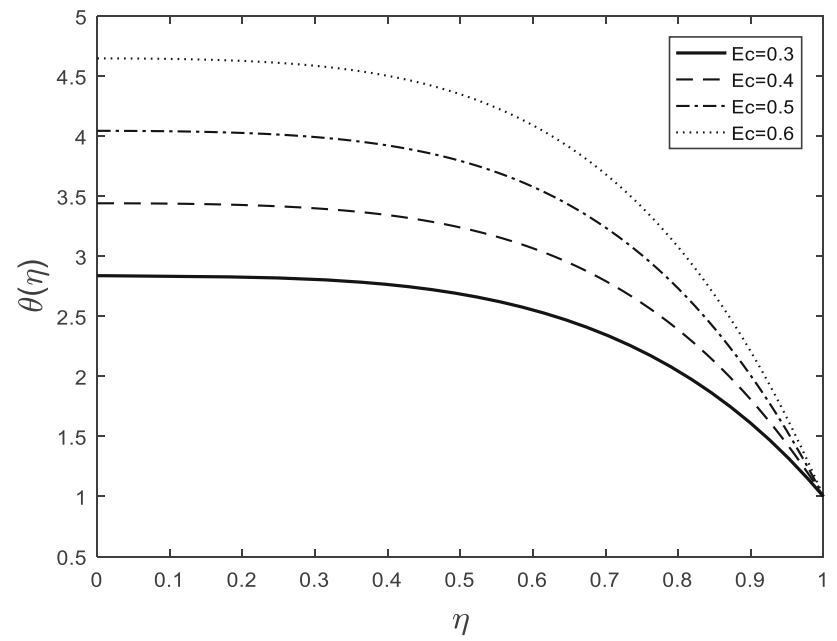

Figure 18. Influence of $E c$ on $\theta(\eta)$ for fixed $\beta=0.1, S=H a=$ $D u=S r=0.5, R=Q=K r=0.1, P r=S c=0.7$ and $\delta=0.1$.

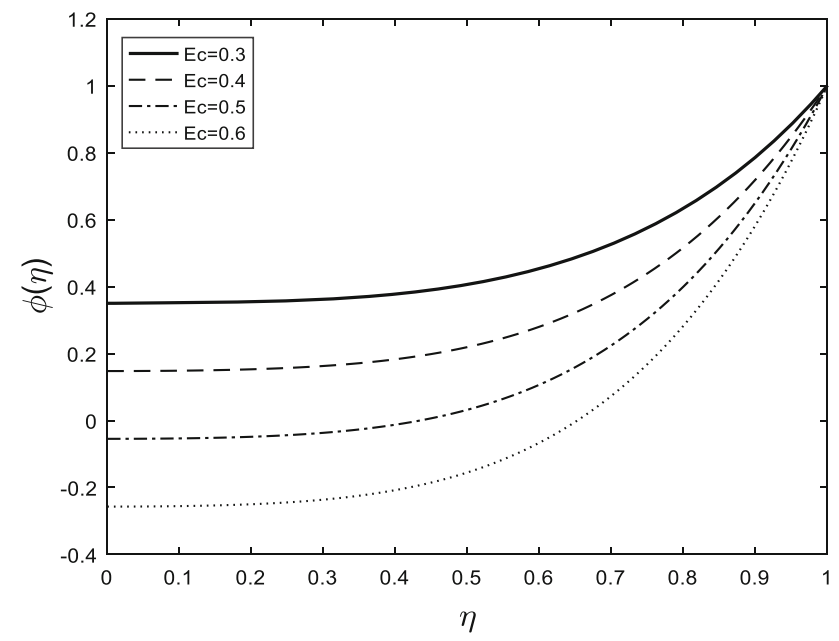

Figure 19. Impact of $E c$ on $\phi(\eta)$ for fixed $\beta=0.1, S=H a=$ $D u=S r=0.5, R=Q=K r=0.1, P r=S c=0.7$ and $\delta=0.1$.

(vii) Impact of $\mathrm{Pr}$ on temperature and concentration profiles

The influence of $\operatorname{Pr}$ on $\theta$ and $\phi$ profiles is portrayed in figures 20 and 21, respectively. From these figures it is noticed that thermal field increases for enhanced $\mathrm{Pr}$ values whereas concentration field is diminished for magnifying values of $\operatorname{Pr}$. Due to the presence of dissipation effects, the temperature field increases in the flow region. Further, thermal boundary layer thickness decays [15] for magnifying values of $\mathrm{Pr}$. This decay is because increased $\mathrm{Pr}$ values greatly suppress the thermal diffusivity, which in turn decreases the thermal boundary layer thickness. Generally, it is known that, $\operatorname{Pr}<1$ is associated with the liquid materials with low viscosity and high thermal conductivity whereas $\operatorname{Pr}>1$ corresponds to the high-viscosity materials like oils, etc. More clearly, temperature field acts like an increasing function of Prandtl number. Also, figure 21

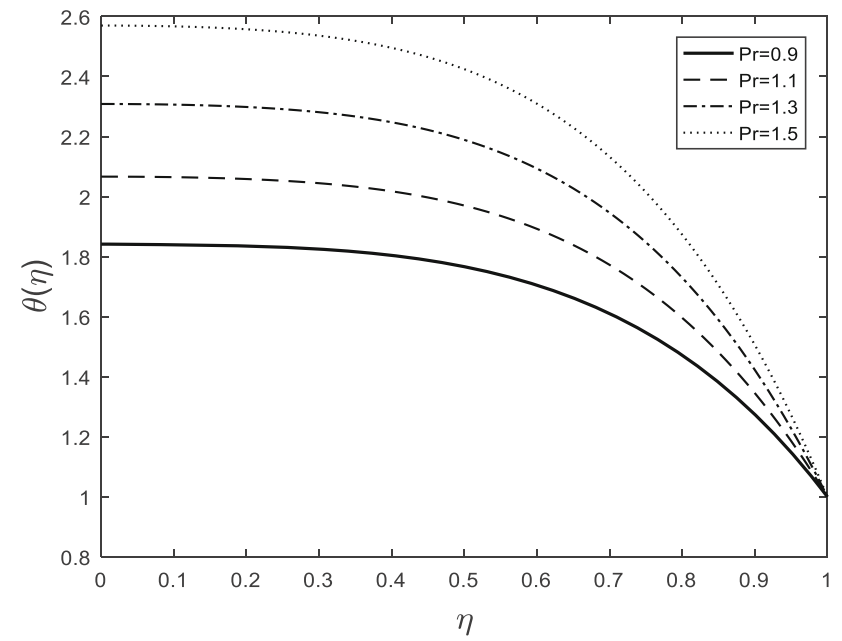

Figure 20. Effect of $\operatorname{Pr}$ on $\theta(\eta)$ for fixed $\beta=0.1, S=H a=$ $D u=S r=0.5, R=Q=E c=K r=0.1, S c=0.7$ and $\delta=0.1$.

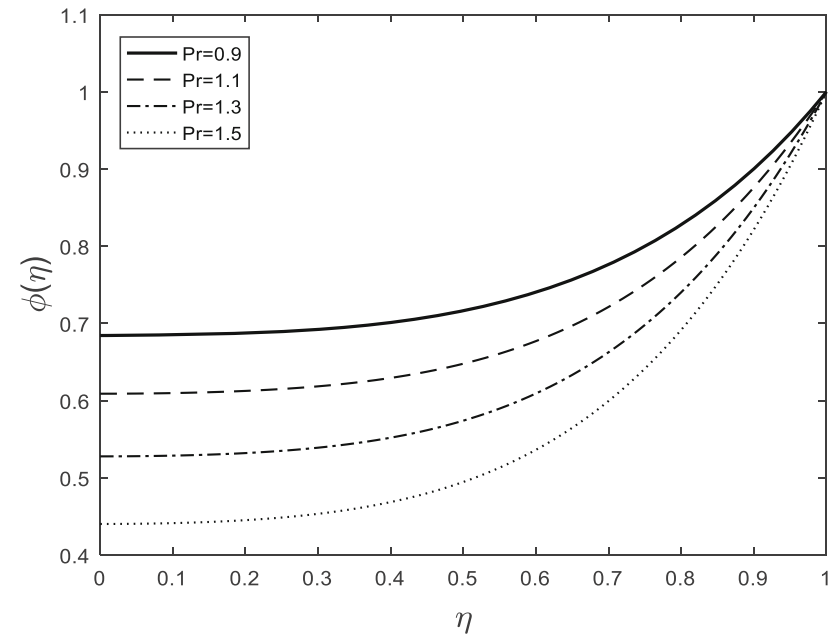

Figure 21. Impact of $\operatorname{Pr}$ on $\phi(\eta)$ for fixed $\beta=0.1, S=H a=$ $D u=S r=0.5, R=Q=E c=K r=0.1, S c=0.7$ and $\delta=0.1$.

shows that, increasing $\operatorname{Pr}$ decreases the concentration field in the flow region.

(viii) Effect of Dufour number $(D u)$ on $\theta$ and $\phi$ profiles

The influence of $D u$ on $\theta$ and $\phi$ fields is shown, respectively, in figures 22 and 23. From figure 22, it is noticed that temperature field increases as $D u$ increases. This is because kinematic viscosity in the flow region decreases. Also, figure 23 demonstrates that a small increment in $D u$ decreases the concentration field. It is clearly observed that temperature field is an increasing function of Dufour number and concentration field is a monotonically decaying function of $\mathrm{Du}$.

(ix) Influence of $K r$ on $\phi$ profile

The impact of $K r$ on concentration profile is shown in figure 24. It is observed from figure 24 that the $\phi$ profile decays for increasing values of $K r>0$. Also, for decreasing values of $K r<0$, the concentration profile increases. In 


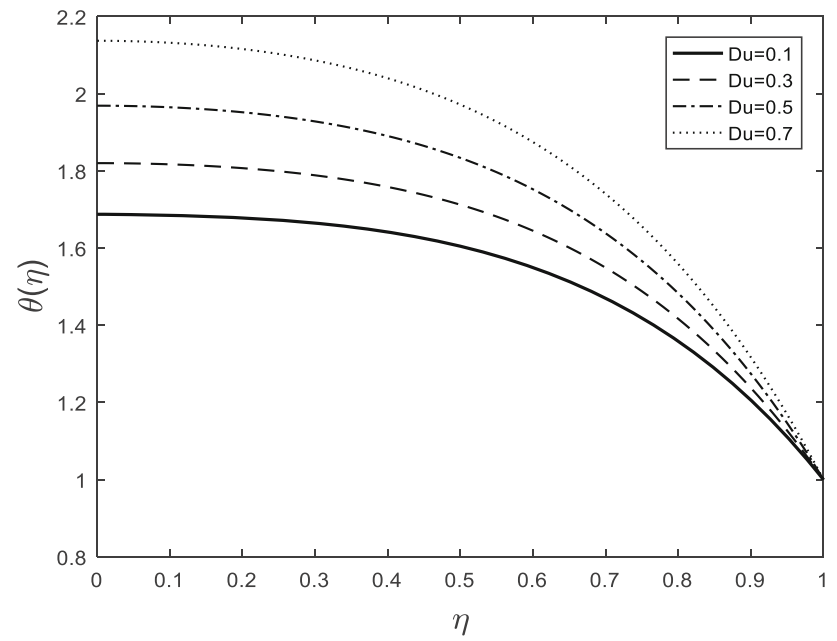

Figure 22. Influence of $D u$ on $\theta(\eta)$ for fixed $S=0.4, \beta=$ $0.2, S r=0.5, H a=R=E c=0.1, Q=0.5, K r=1.6, P r=1.2$, $S c=0.7$ and $\delta=0.01$.

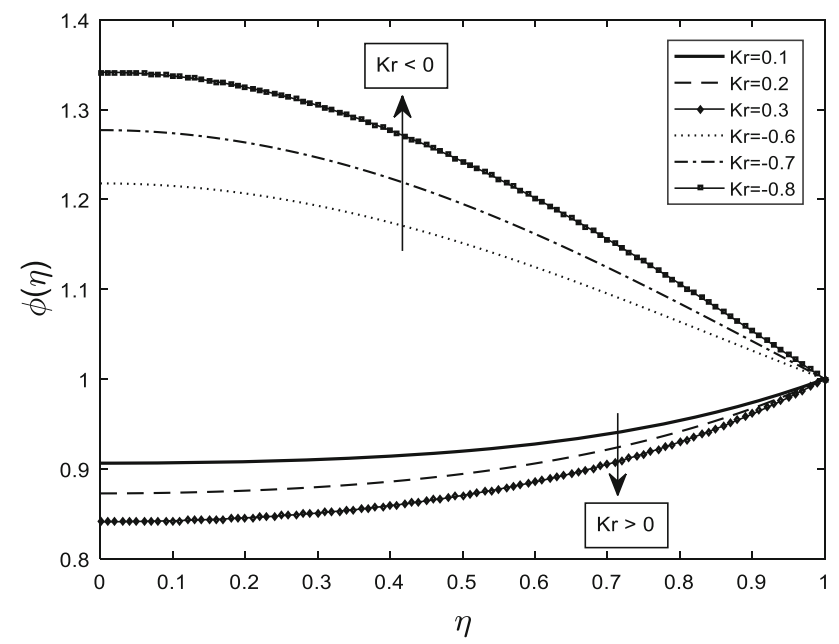

Figure 24. Impact of $K r$ on $\phi(\eta)$ for fixed $S=H a=R=Q=$ $E c=0.1, \beta=D u=S r=0.5, P r=S c=0.7$ and $\delta=0.1$.

many cases, decreased concentration field is observed for destructive chemical reactions [15]. Thus, figure 24 generalizes the results obtained in [15]. Figure 24 clearly illustrates that for $K r>0$, concentration field decreases. This is due to the increased distance between the parallel plates. Further, increased concentration field is observed for $K r<0$ since the distance between plates decreases and causes an increase in the concentration field in the flow region.

(x) Effect $S c$ on $\phi$ profile

The impact of $S c$ on $\phi$ field is depicted in figure 25 . Figure 25 shows that, as $S c$ increases, the concentration profile decays. This is mainly due to the fact that an increment in $S c$ leads to decrease in the molecular diffusion coefficient, which in turn reduces the concentration field in

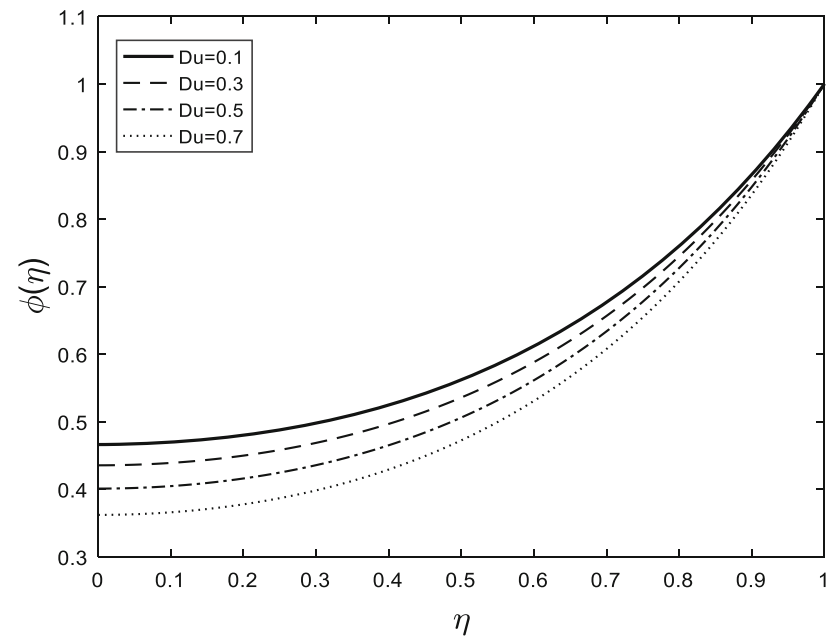

Figure 23. Effect of $D u$ on $\phi(\eta)$ for fixed $S=0.4, \beta=0.2, S r=$ $0.5, H a=R=E c=0.1, Q=0.5, K r=1.6, P r=1.2, S c=0.7$ and $\delta=0.01$.

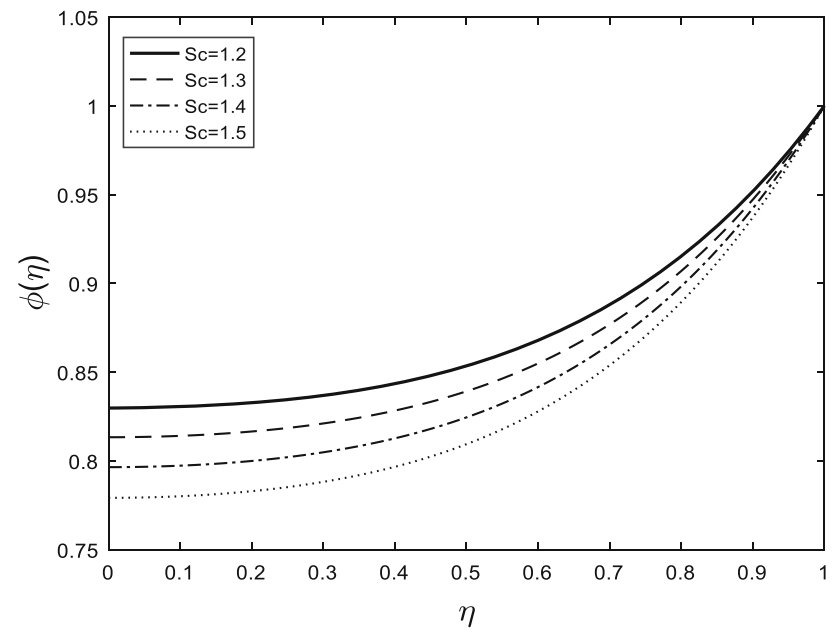

Figure 25. Effect of $S c$ on $\phi(\eta)$ for fixed $S=H a=R=Q=$ $E c=K r=0.1, \beta=D u=S r=0.5, P r=0.7$ and $\delta=0.1$.

the flow region. Further, it is noticed that, the species concentration is high at the wall of the plates and it is less far away from parallel plates. Further, it is worth noting that the $\phi$ field is a monotonically diminishing function of $S c$.

(xi) Effect of Soret number $(\mathrm{Sr})$ on concentration profile

The thermodynamic variations noticed in temperature and concentration profiles with respect to Soret number $(\mathrm{Sr})$ are portrayed in figures 26 and 27 , respectively. It is noticed from figure 26 that the thermal field increases with increase in $S r$. Further, figure 27 shows that, as $S r$ increases, the concentration field decreases in the flow region. This is owing to the fact that an upsurge in $\mathrm{Sr}$ decreases kinematic viscosity of the fluid, which in turn decreases concentration. Also, concentration field behaves like a monotonically decreasing function of $\mathrm{Sr}$. 


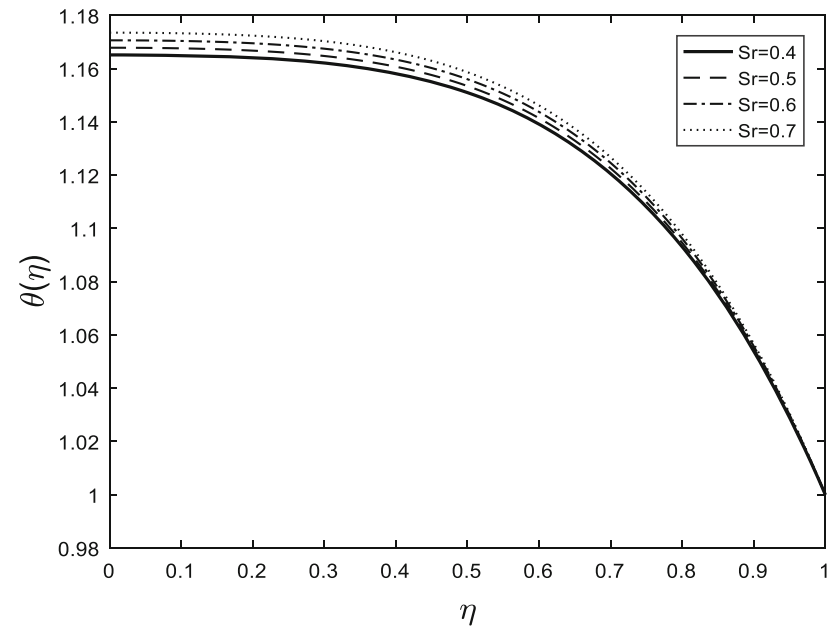

Figure 26. Impact of $S r$ on $\theta(\eta)$ for fixed $S=H a=R=Q=$ $E c=K r=0.1, \beta=D u=0.5, P r=0.7, S c=0.5$ and $\delta=0.1$.

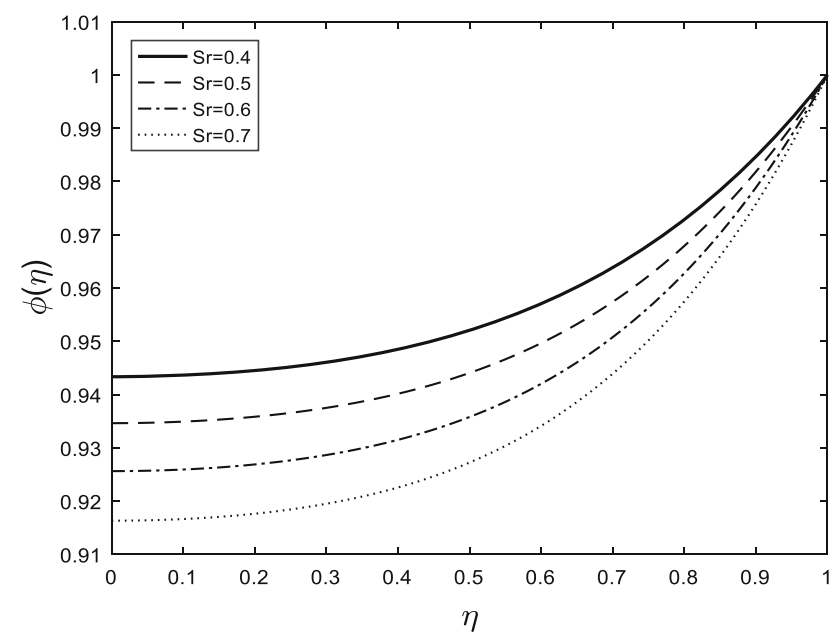

Figure 27. Influence of $S r$ on $\phi(\eta)$ for fixed $S=H a=R=Q=$ $E c=K r=0.1, \beta=D u=0.5, P r=0.7, S c=0.5$ and $\delta=0.1$.

\section{Physical quantities of engineering interest}

The physical quantities such as local skin-friction coefficient, Nusselt and Sherwood numbers play a key role in the field of mechanical and aerospace engineering sciences. Also, in the recent times, the momentum, heat and mass transfer coefficients have a large number of applications in thermodynamic industries. To this end, in the present study, an attempt is made to investigate the thermodynamic behaviour of these quantities in detail and the computational values of momentum transfer coefficient $\left(F^{\prime \prime}(1)\right)$, Nusselt $\left(\theta^{\prime}(1)\right)$ and Sherwood $\left(\phi^{\prime}(1)\right)$ numbers are presented in tables 3, 4 and 5, respectively. Here, the numerical values of skin-friction coefficient, Nusselt and Sherwood numbers are calculated using the following standard definitions:
Table 3. Momentum transport coefficient for different values of $S, \beta$ and $H a \quad$ when $\quad R=Q=E c=K r=0.2, D u=S r=\delta=$ $0.5, \operatorname{Pr}=S c=0.7$ and $\eta=1$.

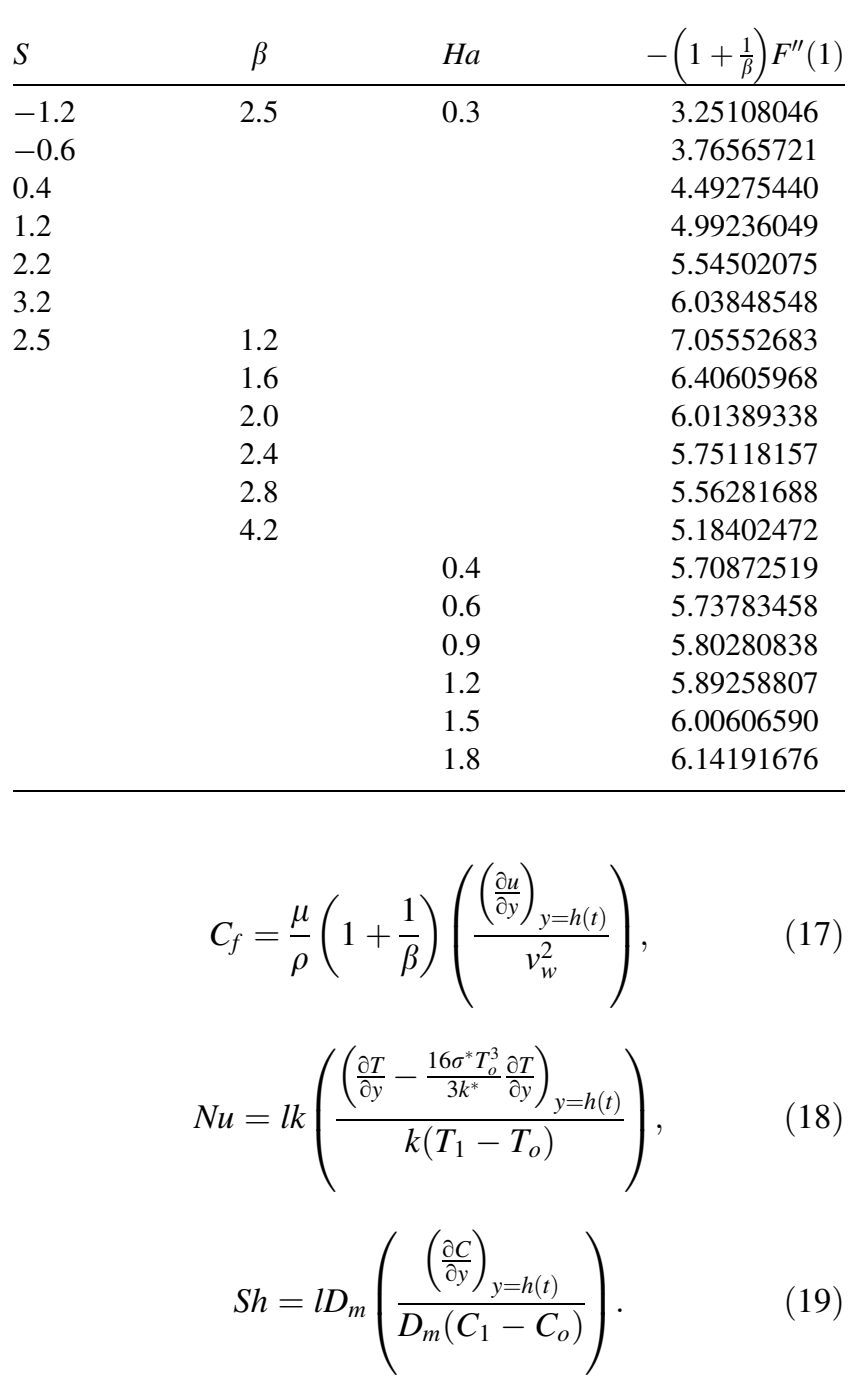

Further, for easier numerical computation purpose, the dimensional equations (17)-(19) are converted to the dimensionless form utilizing Eq. (8) and the resulting equations are given as follows:

$$
\begin{gathered}
\frac{l^{2}}{x^{2}(1-\alpha t)} \operatorname{Re}_{x} C_{f}=\left(1+\frac{1}{\beta}\right) F^{\prime \prime}(1), \\
\sqrt{(1-\alpha t)} N u=-\left(1+\frac{4}{3} R\right) \theta^{\prime}(1), \\
\sqrt{(1-\alpha t)} S h=-\phi^{\prime}(1) .
\end{gathered}
$$

Tables 3, 4 and 5 are presented to describe the thermodynamic behaviour of $F^{\prime \prime}(1), \theta^{\prime}(1)$ and $\phi^{\prime}(1)$ in the flow region for the different values of physical parameters. The impacts of $S, \beta$ and $\mathrm{Ha}$ on skin-friction coefficient is illustrated in the table 3 . It is observed from table 3 that the magnitude of $F^{\prime \prime}(1)$ decays for $S>0$, and it is magnified 
Table 4. Heat transport coefficient for different values of $S, R, Q$ and $D u$ when $H a=1.5, \beta=S r=0.5, P r=S c=0.7, E c=0.2$, $K r=0.2, \delta=0.1$ and $\eta=1$.

\begin{tabular}{|c|c|c|c|c|}
\hline$S$ & $R$ & $Q$ & $D u$ & $-\left(1+\frac{4}{3} R\right) \theta^{\prime}(1)$ \\
\hline-1.2 & 0.3 & 0.3 & 0.2 & 1.45365837 \\
\hline-0.6 & & & & 1.59093138 \\
\hline 0.4 & & & & 1.83282374 \\
\hline 1.2 & & & & 2.03783860 \\
\hline 2.2 & & & & 2.30876671 \\
\hline 3.2 & & & & 2.59701281 \\
\hline \multirow[t]{15}{*}{2.5} & 0.1 & & & 2.44016100 \\
\hline & 0.2 & & & 2.41406132 \\
\hline & 0.4 & & & 2.37652594 \\
\hline & 0.5 & & & 2.36257946 \\
\hline & 0.6 & & & 2.35083318 \\
\hline & & 0.1 & & 1.89029498 \\
\hline & & 0.2 & & 2.13013386 \\
\hline & & 0.4 & & 2.68415754 \\
\hline & & 0.5 & & 3.00782432 \\
\hline & & 0.6 & & 3.37108903 \\
\hline & & & 0.1 & 2.34427662 \\
\hline & & & 0.3 & 2.44410771 \\
\hline & & & 0.4 & 2.49662277 \\
\hline & & & 0.5 & 2.55099229 \\
\hline & & & 0.6 & 2.60731545 \\
\hline
\end{tabular}

Table 5. Mass transport coefficient for different values of $S, S r$ and $K r$ when $P r=S c=0.7, \beta=H a=D u=\delta=0.5, R=$ $Q=E c=0.2$ and $\eta=1$.

\begin{tabular}{lcrr}
\hline$S$ & $S r$ & $K r$ & \multicolumn{1}{c}{$\phi^{\prime}(1)$} \\
\hline-1.2 & 0.2 & 0.1 & 0.26949810 \\
-0.6 & & & 0.27176008 \\
0.4 & & & 0.27705697 \\
1.2 & & & 0.28222520 \\
2.2 & & & 0.28941028 \\
3.2 & & & 0.29707946 \\
4.6 & 0.4 & & 0.30820328 \\
2.5 & 0.6 & & 0.53909446 \\
& 0.8 & & 0.80606421 \\
& 1.0 & & 1.09497363 \\
& 1.2 & -1.2 & 1.40861744 \\
& & -0.7 & 1.75028091 \\
& & 0.8 & -0.77788286 \\
& & 1.8 & 0.27575934 \\
& & 2.2 & 1.04992465 \\
& & 2.8 & 1.18220362 \\
& & & 1.36080413 \\
& & &
\end{tabular}

for $S<0$. Also, it is remarked that, the magnifying values of $\beta$ decrease the magnitude of wall shear stress. Further, magnitude of skin-friction coefficient increases for increasing $\mathrm{Ha}$ values.

Table 4 illustrates the behaviour of heat transfer rate $\left(\theta^{\prime}(1)\right)$ under the influence of different flow parameters such as squeezing and Dufour numbers, radiation and heat generation or absorption parameters. It is noticed from table 4 that the magnitude of $\theta^{\prime}(1)$ upsurges for increasing values of $S$. Also, magnitude of heat transfer rate decreases for magnifying values of thermal radiation parameter in the flow region. Also, the magnitude of $\theta^{\prime}(1)$ increases for increasing values of $Q$ and $D u$.

Further, table 5 illustrates the behaviour of mass transfer rate $\left(\phi^{\prime}(1)\right)$ under the influence of squeezing parameter, Soret number and $K r$. It is clearly remarked that the magnitude of $\phi^{\prime}(1)$ increases for increasing values of squeezing number. Also, it is observed that, the magnitude of $\phi^{\prime}(1)$ increases for increasing values of Soret number in the flow region. Further, mass transfer rate is suppressed for increasing values of $K r$ (i.e., $K r>0$ ) and this is owing to the constructive behaviour of the considered chemical reaction. However, $\phi^{\prime}(1)$ increases for decreasing values of $K r$ (i.e., $K r<0$ ) and this is mainly owing to the destructive nature of the chemical reaction.

\section{Conclusions}

The present numerical study investigates the heat and mass characteristics of time-dependent two-dimensional MHD squeezing flow of Casson fluid between two parallel plates under the influence of Soret and Dufour effects with radiation and Joule dissipation impacts in the presence of homogeneous first order chemical reaction. Due to the movement of the parallel plates, flow occurs. The coupled 
nonlinear fluid flow equations are derived taking into account of Casson fluid model and these equations are solved by using RK-SM and bvp4c techniques. The thermodynamic behaviour of physical parameters is illustrated through the graphs and tables. Numerical simulations are carried out for different sets of flow parameters. Following important observations are made from the present study:

i. Velocity field is suppressed when $S>0$ and it is magnified for $S<0$.

ii. Temperature profile is diminished for $S>0$ and enhanced for $S<0$.

iii. Temperature field is a decreasing function of $\beta$. However, velocity field is enhanced for magnifying values of $\beta$.

iv. Velocity and concentration fields decrease for increasing values of Hartmann number.

v. Temperature field decreases for increasing values of thermal radiation parameter.

vi. Temperature profile is enhanced for $Q>0$ and it is suppressed for $Q<0$.

vii. An increment in Dufour number increases the temperature field.

viii. Concentration field is eventually suppressed for magnifying values of $S r$ and $S c$.

ix. Concentration profile decreases for $K r>0$ and it increases for $K r<0$.

\section{Acknowledgements}

The authors wish to express their gratitude to the reviewers who highlighted important areas for improvement in this earlier draft of the article. Their suggestions have served specifically to enhance the clarity and depth of the interpretation of results in the revised manuscript. One of the author, Usha Shankar, wishes to thank Karnataka Power Corporation Limited, Raichur Thermal Power Station, Shaktinagar, for the encouragement.

\section{List of symbols}

$S \quad$ squeezing number

$\mathrm{Ha}$ Hartmann number

Ec Eckert number

$R \quad$ radiation parameter

$Q \quad$ heat generation or absorption parameter

$Q^{*} \quad$ volumetric heat generation or absorption coefficient

Pr Prandtl number

Sc Schmidt number

$\mathrm{Sr} \quad$ Soret number

$\mathrm{Du} \quad$ Dufour number

$\mathrm{Kr} \quad$ chemical reaction parameter

$\kappa \quad$ thermal conductivity $\left(\mathrm{W} \mathrm{m} \mathrm{m}^{-1} \mathrm{~K}^{-1}\right.$ )

$k^{*} \quad$ absorption coefficient $\left(\mathrm{m}^{-1}\right)$

$D_{m} \quad$ coefficient of mass diffusion
$C_{f} \quad$ skin-friction coefficient

$\mathrm{Nu} \quad$ Nusselt number

Sh Sherwood number

$R e_{x} \quad$ Reynolds number

$p \quad$ pressure $(\mathrm{Pa})$

$C_{p} \quad$ specific heat capacity at constant pressure $\left(\mathrm{J} \mathrm{kg}^{-1} \mathrm{~K}^{-1}\right.$ )

$C_{s} \quad$ specific heat capacity at constant concentration

$B_{0} \quad$ uniform magnetic field

$l \quad$ initial distance between the parallel plates (m)

$T$ dimensional fluid temperature $(\mathrm{K})$

$T_{w} \quad$ wall temperature (K)

$T_{\infty} \quad$ ambient fluid temperature (K)

C dimensional fluid concentration $\left(\mathrm{mol} / \mathrm{m}^{3}\right)$

$C_{w} \quad$ wall concentration $\left(\mathrm{mol} / \mathrm{m}^{3}\right)$

$C_{\infty} \quad$ ambient fluid concentration $\left(\mathrm{mol} / \mathrm{m}^{3}\right)$

$k_{1} \quad$ chemical reaction coefficient

$u, v \quad$ dimensional velocity components along $x, y$ directions $\left(\mathrm{m} \mathrm{s}^{-1}\right)$

$F^{\prime}, F$ non-dimensional velocity components along axial and radial directions

\section{Greek symbols}

$\beta \quad$ Casson fluid parameter

$\sigma \quad$ electrical conductivity $\left(\mathrm{s} \mathrm{m}^{-1}\right)$

$\sigma^{*} \quad$ Stefan-Boltzmann constant $\left(\mathrm{W} \mathrm{m}^{-2} \mathrm{~K}^{-4}\right)$

$\eta \quad$ similarity variable

$\theta \quad$ dimensionless temperature

$\phi \quad$ dimensionless concentration

$\rho \quad$ fluid density $\left(\mathrm{kg} \mathrm{m}^{-3}\right)$

$\mu$ dynamic viscosity $\left(\mathrm{Ns} \mathrm{m}^{-2}\right)$

$\alpha \quad$ characteristic parameter of the squeezing motion of the plate $\left(\mathrm{s}^{-1}\right)$

$v \quad$ kinematic viscosity of the fluid $\left(\mathrm{m}^{2} \mathrm{~s}^{-1}\right)$

\section{References}

[1] Lawal A and Kalyon D M 1998 Squeezing flow of viscoplastic fluids subject to wall slip. Polym. Eng. Sci. 38(11): 1793-1804

[2] Maki E R, Kuzma D C and Donnelly R J 1966 Magnetohydrodynamic lubrication flow between parallel plates. $J$. Fluid Mech. 26(3): 537-543

[3] Dorier C and Tichy J 1992 Behaviour of a Bingham-like viscous fluid in lubrication flows. J. Non-Newton. Fluid Mech. 45: 291-310

[4] Mohsin B B, Ahmed N, Adnan, Khan U and Mohyud-Din S T 2017 A bio-convection model for a squeezing flow of nanofluid between parallel plates in the presence of gyrotactic microorganisms. Eur. Phys. J. Plus 132 (187): 1-12

[5] Collyer A A and Clegg D W 1998 Rheological measurement, 2nd ed. London, UK: Chapman \& Hali

[6] Khan H, Qayyum M, Khan O and Ali M 2016 Unsteady squeezing flow of Casson fluid with magneto-hydrodynamic effect and passing through porous medium. Math. Probl. Eng. https://doi.org/10.1155/2016/4293721 
[7] Stefan M J 1874 Versuchüber die scheinbare adhesion. Sitzungsber Sächs Akad Wiss Wein. Math-Nat Wiss Kl 69: 713-721

[8] Reynolds O 1886 On the theory of lubrication. Philos. Trans. R. Soc. Lond. 177: 157-234

[9] Archibald F R 1956 Load capacity and time relations for squeeze films. Trans. ASME 78: 231-245

[10] Grimm G J 1976 Squeezing flows of Newtonian liquid films: an analysis includes the fluid inertia. Appl. Sci. Res. 32(2): 149-166

[11] Wolfe W A 1965 Squeeze film pressures. Appl. Sci. Res. 14: 77-90

[12] Kuzma D C 1968 Fluid inertia effects in squeeze films. Appl. Sci. Res. 18: 15-20

[13] Tichy J A and Winer W O 1970 Inertial considerations in parallel circular squeeze film bearings. J. Lubr. Technol. 92: 588-592

[14] Jackson J D 1962 A study of squeezing flow. Appl. Sci. Res. 11: $148-152$

[15] Mustafa M, Hayat T and Obaidat S 2012 On heat and mass transfer in the unsteady squeezing flow between parallel plates. Meccanica 47: 1581-1589

[16] Siddiqui A M, Irum S and Ansari A R 2008 Unsteady squeezing flow of a viscous MHD fluid between parallel plates, a solution using the homotopy perturbation method. Math. Model. Anal. 13(4): 565-576

[17] Domairry G and Aziz A 2009 Approximate analysis of MHD squeeze flow between two parallel disks with suction or injection by homotopy perturbation method. Math. Probl. Eng. https://doi.org/10.1155/2009/603916

[18] Duwairi H M, Tashtoush B and Damseh R A 2004 On heat transfer effects of a viscous fluid squeezed and extruded between two parallel plates. Heat Mass Transf. 41(2): 112-117

[19] Mahmood M, Asghar S and Hossain M A 2007 Squeezed flow and heat transfer over a porous surface for viscous fluid. Heat Mass Transf. 44: 165-173

[20] Tashtoush B, Tahat M and Probert D 2001 Heat transfers and radial flows via a viscous fluid squeezed between two parallel disks. Appl. Energy 68: 275-288

[21] Muhaimin, Kandasamy R and Hashim I 2010 Effect of chemical reaction, heat and mass transfer on nonlinear boundary layer past a porous shrinking sheet in the presence of suction. Nucl. Eng. Des. 240: 933-939

[22] Bahadir A R and Abbasov T 2011 A numerical approach to hydromagnetic squeezed flow and heat transfer between two parallel disks. Ind. Lubr. Tribol. 63(2): 63-71

[23] Weaver J A and Viskanta R 1991 Natural convection due to horizontal temperature and concentration gradients-2. Species interdiffusion, Soret and Dufour effects. Int. J. Heat Mass Transf. 34(12): 3121-3133
[24] Raju C S K and Sandeep N 2016 Heat and mass transfer in MHD non-Newtonian bio-convection flow over a rotating cone/plate with cross diffusion. J. Mol. Liq. 215: 115-126

[25] Sulochana C, Payad S S and Sandeep N 2015 Non-uniform heat source or sink effect on the flow of 3D Casson fluid in the presence of Soret and thermal radiation. Int. J. Eng. Res. Afr. 20: 112-129

[26] Nawaz M, Hayat T and Alsaedi A 2012 Dufour and Soret effects on MHD flow of viscous fluid between radially stretching sheets in porous medium. Appl. Math. Mech. Engl. Ed. 33(11): 1403-1418

[27] Ojjela O and Kumar N N 2016 Unsteady MHD mixed convective flow of chemically reacting and radiating couple stress fluid in a porous medium between parallel plates with Soret and Dufour effects. Arab. J. Sci. Eng. 41: 1941-1953

[28] Khan U, Ahmed N and Mohyud-Din S T 2016 Thermodiffusion, diffusion-thermo and chemical reaction effects on MHD flow of viscous fluid in divergent and convergent channels. Chem. Eng. Sci. 141(17): 17-27

[29] Khan S I, Mohyud-Din S T and Mohsin B B 2017 Thermodiffusion and diffuso-thermo effects on MHD squeezing flow between parallel disks. Surf. Rev. Lett. 24(2): 1750022-10

[30] Ahmed N, Khan U, Khan S I, Bano S and Mohyud-Din S T 2017 Effects on magnetic field in squeezing flow of a Casson fluid between parallel plates. J. King Saud Univ. Sci. 29: 119-125

[31] Mohyud-Din S T, Usman M, Wang W and Hamid M 2017 A study of heat transfer analysis for squeezing flow of a Casson fluid via differential transform method. Neural Comput. Appl. 30(10): 3253-3264

[32] Cebeci T and Bradshaw P 1984 Physical and computational aspects of convective heat transfer. New York, USA: Springer-Verlag

[33] Kiusalaas J 2005 Numerical methods in engineering with MATLAB. UK: Cambridge University Press

[34] Butcher J C 2016 Numerical methods for ordinary differential equations. UK: John Wiley \& Sons

[35] Butcher J C 1975 A stability property of implicit RungeKutta methods. BIT Numer. Math. 15: 358-361

[36] Butcher J C and Wanner G 1996 Runge-Kutta methods: some historical notes. Appl. Numer. Math. 22: 113-151

[37] Houwen P J V D, Wolkenfelt P H M and Baker C T H 1981 Convergence and stability analysis for modified Runge-Kutta methods in the numerical treatment of second-kind Volterra integral equations. IMA J. Numer. Anal. 1: $303-328$

[38] Debrabant K and Strehmel K 2005 Convergence of RungeKutta methods applied to linear partial differential-algebraic equations. Appl. Numer. Math. 53: 213-229 